\title{
os \\ Interferometric signature of non-Abelian anyons
}

\author{
Waheb Bishara, ${ }^{1}$ Parsa Bonderson, ${ }^{2}$ Chetan Nayak, ${ }^{2,3}$ Kirill Shtengel, ${ }^{4,5}$ and J. K. Slingerland ${ }^{6,7}$ \\ ${ }^{1}$ Department of Physics, California Institute of Technology, Pasadena, California 91125, USA \\ ${ }^{2}$ Microsoft Research, Station Q, Elings Hall, University of California, Santa Barbara, California 93106, USA \\ ${ }^{3}$ Department of Physics, University of California, Santa Barbara, California 93106, USA \\ ${ }^{4}$ Department of Physics and Astronomy, University of California at Riverside, Riverside, California 92507, USA \\ ${ }^{5}$ Institute for Quantum Information, California Institute of Technology, Pasadena, California 91125, USA \\ ${ }^{6}$ School of Theoretical Physics, Dublin Institute for Advanced Studies, 10 Burlington Road, Dublin, Ireland \\ ${ }^{7}$ Department of Mathematical Physics, National University of Ireland, Maynooth, Ireland
}

(Received 27 May 2009; revised manuscript received 13 August 2009; published 5 October 2009)

\begin{abstract}
We consider the tunneling current through a double point-contact Fabry-Pérot interferometer such as used in recent experimental studies of the fractional quantum Hall plateau at filling fraction $\nu=5 / 2$. We compare the predictions of several different models of the state of the electrons at this plateau: the Moore-Read, antiPfaffian, $\mathrm{SU}(2)_{2} \mathrm{NAF}, K=8$ strong pairing, and $(3,3,1)$ states. All of these predict the existence of charge $e / 4$ quasiparticles, but the first three are non-Abelian while the last two are Abelian. We give explicit formulas for the scaling of charge $e / 2$ and charge $e / 4$ quasiparticle contributions to the current as a function of temperature, gate voltage, and distance between the two point contacts for all three models. Based on these, we analyze several possible explanations of two phenomena reported for recent experiments by Willett et al., namely, halving of the period of the observed resistance oscillations with rising temperature and alternation between the same two observed periods at low temperatures as the area of the interference loop is varied with a side gate. We conclude that the most likely explanation is that the observed alternation is due to switching between even and odd numbers of charge $e / 4$ quasiparticles enclosed within the loop as a function of side-gate voltage, which is a clear signature of the presence of non-Abelian anyons. However, there are important features of the data which do not have a simple explanation within this picture. We suggest further experiments which could help rule out some possible scenarios. We make the corresponding predictions for future tunneling and interference experiments at the other observed second Landau level fractional quantum Hall states.
\end{abstract}

DOI: 10.1103/PhysRevB.80.155303

PACS number(s): 71.10.Pm, 73.43.Jn, 05.30.Pr

\section{INTRODUCTION}

The observation ${ }^{1,2}$ of a fractional quantum Hall (FQH) state at $\nu=5 / 2$ and suggestion ${ }^{3}$ that the Moore-Read Pfaffian (MR) state ${ }^{4-6}$ might occur at this filling fraction gave the first real indication that non-Abelian topological phases of matter might actually occur in nature. The striking feature of such new phases is that they possess quasiparticle excitations with exotic non-Abelian braiding statistics. ${ }^{7-13}$ This property makes non-Abelian topological phases appealing for their potential use as intrinsically fault-tolerant media for quantum information processing. ${ }^{14-21}$

Recent experimental studies of transport through a point contact in $\mathrm{FQH}$ systems at $\nu=5 / 2$ gave evidence that there are charge $e / 4$ quasiparticles in this state 22 and found that the dependence of the current on voltage and temperature is most consistent ${ }^{23}$ with two particular non-Abelian models: the anti-Pfaffian $(\overline{\mathrm{Pf}})$ state $^{24,25}$ and the SU(2) 2 NAF (nonAbelian FQH) state. ${ }^{26,27}$ However, these results are not conclusive because the $(3,3,1)$ state, ${ }^{28}$ which is Abelian, also supports charge $e / 4$ quasiparticles. It is also roughly consistent with the voltage and temperature dependence of tunneling found in Ref. 23 and, in any case, one might expect nonuniversal physics to have a significant effect on the observed dependence. Thus, there is a glaring need for experiments which directly probe the braiding statistics of quasiparticles.

In order to probe braiding statistics in FQH systems, one can use a double point-contact interferometer as proposed in
Ref. 29 for Abelian states and later considered for the $\nu$ $=5 / 2$ state in Refs. 30-33. Such interferometers can play a crucial role in properly identifying which phase a FQH state is in by providing information about the topological $S$ matrix, a mathematical quantity related to the braiding statistics that is strongly characteristic of the topological order (for more details, see Ref. 34). Interferometers are also important for the implementation of topological quantum computation $^{14,16}$ because they can be used for the topological charge measurements necessary for readout of qubits ${ }^{31}$ and, through adroit manipulation, can even be used to implement computational gates. ${ }^{35,36}$ Fortunately, there have been recent advances in realizing quantum Hall interferometers at integer filling 37,38 and fractional filling in the lowest Landau level. ${ }^{39,40}$ Even more recently, double point-contact interferometers have been experimentally implemented for the $\nu$ $=5 / 2$ FQH state. ${ }^{41-44}$

In this paper, we study the signatures of non-Abelian statistics which can be seen in a double point-contact interferometer and discuss other effects which can mimic these signatures. We propose further experiments which can help disentangle the effects of non-Abelian statistics from Coulomb blockade and disorder physics. Our discussion may be relevant to the following prediction attributed to Shtengel in Ref. 45: "With luck, we might see a non-Abelian interferometer within a year." The paper is structured as follows: in Sec. II, we describe the basic features of the experiment of Willett et $a l .{ }^{42-44}$ In Sec. III, we explain three different inter- 


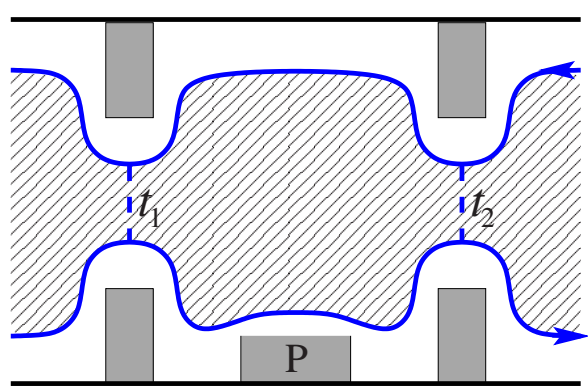

FIG. 1. (Color online) A double point-contact interferometer. Edge quasiparticles tunnel at two point contacts with amplitudes $t_{1}$ and $t_{2}$, respectively. The interferometry area is changed by applying a voltage $V_{s}$ to a plunger gate $P$ that depletes the 2DEG beneath it. Quantum interference between the two paths manifests an observable signature of the Aharonov-Bohm effect and the braiding statistics (of the edge quasiparticle with the bulk quasiparticles in the central interferometry region) in the oscillation patterns of the tunneling current when the area is changed.

pretations of this experiment: (a) non-Abelian interferometry, (b) Coulomb blockade, and (c) possible explanations loosely grouped together because they depend on nonlinear dependence of the interferometer area on the side-gate voltage $V_{s}$. In Sec. IV, we criticize each of these three interpretations. We argue that, while all three interpretations have problems, the problems with (b) and (c) are more serious and these explanations are less likely to be correct. In Sec. V, we propose further experiments which might further strengthen or rule out interpretation (a). In Sec. VI, we comment on the implications of this experiment for topological quantum computation assuming that the non-Abelian interference interpretation is correct. In the two appendixes, we give predictions for interference experiments at other suspected nonAbelian fractions and we argue that the bare backscattering amplitude for $e / 4$ quasiparticles should be much larger than that for $e / 2$ quasiparticles.

\section{EXPERIMENT}

In recent experiments, Willett et al. ${ }^{42-44}$ measured the current through a double point-contact device depicted schematically in Fig. 1. As a function of magnetic field $B$, the longitudinal resistance $R_{L}$ of the device has prominent minima at roughly the $B$ values at which the $\nu=2,7 / 3,5 / 3$, and $5 / 2$ quantum Hall states occur in the bulk (near, but not at, the point contacts). At the minima corresponding to $\nu$ $=5 / 2$ and $7 / 3$, the longitudinal resistance is $R_{L}$ $\simeq 200-300 \Omega$, while at $\nu=2$ and $5 / 3$ it is $R_{L}<50 \Omega$. There are small oscillations with $B$ on top of these large features, but these were not the focus of the experiment since changing the magnetic field can change both the flux enclosed and, possibly, the quasiparticle number, thereby making it difficult to isolate the effect of braiding statistics. Instead, a side-gate voltage is varied as shown in Fig. 1. As the side-gate voltage $V_{s}$ is varied, $R_{L}$ oscillates with an amplitude of roughly $2 \Omega$.

The period of the oscillations, $\Delta V_{s}$, is larger at $\nu=5 / 3$ and $7 / 3$ than at $\nu=2$. This was interpreted in the following way: it was assumed that the principle effect of varying the side-gate voltage is to change the area of the interference loop between the two point contacts and that they are related linearly by $\Delta A=c \Delta V_{s}$, where $c$ is essentially constant, even between different filling fractions. Thus, the oscillations are hypothesized to be due to the Aharonov-Bohm (AB) effect, which implies a period $\Delta A=\left(e / e^{*}\right) \Phi_{0} / B$, where $e^{*}$ is the charge of the tunneling quasiparticle and $-e$ is the electron charge, and $\Phi_{0}=h c / e$ is the magnetic flux quantum. Willett et al. ${ }^{43,44}$ analyzed their data to find that the period at $\nu=5 / 3$ and $7 / 3$, normalized by the corresponding magnetic fields, is three times larger than at $\nu=2: \quad(\Delta A)_{5 / 3} B_{5 / 3} \approx(\Delta A)_{7 / 3} B_{7 / 3}$ $\approx 3(\Delta A)_{2} B_{2}$. Thus, they interpret their findings as evidence that $e^{*} / e=1 / 3$ at $\nu=5 / 3,7 / 3$, assuming that the oscillation period at $\nu=2$ reflects interference of ordinary electrons. At $\nu=5 / 2$, two types of behavior are seen at $25 \mathrm{mK}$. In some regions, which we will call type I, $(\Delta A)_{5 / 2}^{\mathrm{I}} B_{5 / 2} \approx 4(\Delta A)_{2} B_{2}$. In the regions of type II, $(\Delta A)_{5 / 2}^{\mathrm{II}} B_{5 / 2} \approx 2(\Delta A)_{2} B_{2}$. At 150 $\mathrm{mK}$, only one behavior is seen: $(\Delta A)_{5 / 2}^{\mathrm{II}} B_{5 / 2} \approx 2(\Delta A)_{2} B_{2}$. The type of oscillations observed for a region of $V_{s}$ were found to be reproducible throughout multiple scans over the period of 7 days. ${ }^{44}$ The type I oscillations in a given region sometimes exhibited a roughly $\pi$ phase shift from one scan to another. In the next section, we discuss several possible explanations for the occurrence of these two periods at $25 \mathrm{mK}$ and the disappearance of one of them at higher temperatures at $\nu$ $=5 / 2$.

\section{INTERPRETATIONS}

\section{A. Non-Abelian interference}

At first glance, these experimental results appear to be dramatically consistent with the predicted behavior of the proposed non-Abelian $\nu=5 / 2 \mathrm{FQH}$ states, particularly with that of the MR, $\overline{\mathrm{Pf}}$, and $\mathrm{SU}(2)_{2} \mathrm{NAF}$ states, all of which have a non-Abelian fundamental quasihole with charge $e / 4$. The basic assumption is that as one changes the area of the interferometry region, one also occasionally changes the number $n_{q}$ of charge $e / 4$ quasiholes contained in the bulk within the interference loop. (For the purposes of this counting, charge $n e / 4$ excitations, where $n \in \mathbb{Z}$, count as $n$ fundamental quasiholes.) Thus, changing the area will cause the edge current to exhibit interference behavior due to the $A B$ effect, modulated by occasional changes in the number of quasiparticles in the loop and their concomitant braiding statistics. The interference term $I_{12}$ of the backscattered current due to lowest order tunneling of $e / 4$ edge quasiholes is predicted to be $\mathrm{b}^{32,33}$

$$
I_{12}^{(e / 4)} \propto \begin{cases}\cos \left(2 \pi \frac{\Phi}{4 \Phi_{0}} \mp \frac{n_{q} \pi}{4}+n_{\psi} \pi\right) & \text { for } n_{q} \text { even } \\ 0 & \text { for } n_{q} \text { odd }\end{cases}
$$

where the - corresponds to the MR and $\mathrm{SU}(2)_{2}$ states and the + to the $\overline{\text { Pf }}$ state; and $n_{\psi}=0$ or 1 , depending on whether the contained quasiparticles are in a collective state corresponding to the $I$ or $\psi$ fusion channel. This interference exhibits the usual $\mathrm{AB}$ oscillations with period $\Delta A=4 \Phi_{0} / B$ corresponding to $e^{*} / e=1 / 4$, but also a striking complete suppression of this term that results from the non-Abelian braiding 
statistics of the edge quasiparticle with the bulk quasiparticles when $n_{q}$ is odd. Thus, as the area of the interferometry region is changed, and bulk quasiparticles enter or exit the interference loop, the non-Abelian states should see $\Delta A$ $=4 \Phi_{0} / B$ oscillations switch on and off, as they do in going from the type I regions to the type II regions in the experiments of Refs. 43 and 44.

The observed reproducibility of oscillation type regions in multiple scans ${ }^{43,44}$ suggests that the bulk $e / 4$ quasiparticles are pinned and do not move on the time scale of the experiment. The observation of the oscillations in a given type I region being shifted by $\pi$ from one scan to the next also agrees with the expected behavior of non-Abelian states. Specifically, the collective state of several quasiparticles, some of which are inside and some outside the interferometry loop, is decohered by the current of edge quasiparticles around the loop. ${ }^{46}$ Hence, depending on the bulk quasiparticles entering or exiting the interferometry loop, the collective state of quasiparticles inside the interferometer may be randomized between $n_{\psi}=0$ and 1 when $n_{q}$ is changed to an even value. (This is the same randomization that gives rise to a non-Abelian signature in the switching noise. ${ }^{47}$ )

There are two sources that could potentially contribute to $\Delta A=2 \Phi_{0} / B$ oscillations in the non-Abelian $\nu=5 / 2$ states. The first is tunneling of the Abelian $e / 2$ edge quasiparticles, ${ }^{48,49}$ which to lowest order gives the interference current ${ }^{29}$

$$
I_{12}^{(e / 2)} \propto \cos \left(2 \pi \frac{\Phi}{2 \Phi_{0}}-\frac{n_{q} \pi}{2}\right) .
$$

The second possibility comes from higher-order tunneling processes where the interference path encircles the interferometry area twice. The resulting double pass interference term in the current coming from second-order tunneling of $e / 4$ edge quasiparticles is ${ }^{48,49}$

$$
I_{1212}^{(e / 4)} \propto\left\{\begin{array}{cl}
\cos \left(2 \pi \frac{\Phi}{2 \Phi_{0}}-\frac{n_{q} \pi}{2}\right) & \text { for } n_{q} \text { even } \\
\cos \left(2 \pi \frac{\Phi}{2 \Phi_{0}}-\frac{n_{q} \pi}{2} \pm \frac{\pi}{2}\right) & \text { for } n_{q} \text { odd }
\end{array}\right.
$$

where the + corresponds to the MR state and the - to the $\overline{\mathrm{Pf}}$ and $\mathrm{SU}(2)_{2}$ states. Of course, this second-order contribution to the tunneling current will typically have much smaller amplitude, since it both incurs an additional tunneling probability factor and doubles the distance over which coherence must be maintained. For the interferometer of Refs. 42-44, the quasiparticle tunneling probability at each point contact is approximately $P_{1} \simeq P_{2} \simeq 0.05$. This estimate is based on the relation ${ }^{50}$

$$
R_{x x}=\frac{h}{e^{2}} \frac{2}{5} \frac{P}{5-P}
$$

for point-contact tunneling of the half-filling edge modes at $\nu=5 / 2$, where $P \simeq P_{1}+P_{2}$ here is roughly the sum of individual tunneling probabilities of the two point contacts, and $R_{x x} \simeq 200 \Omega$ in Refs. 42-44. Furthermore, there will generally be a suppression of the interference oscillation ampli- tudes that results from the loss of coherence, as well as from having unequal tunneling probabilities at the two point contacts (which gives a suppression of $2 \sqrt{P_{1} P_{2}} / P_{1}+P_{2}$ ). Roughly speaking, we can define the suppression factor to be

$$
Q \simeq \max \left(I_{12}\right) /\left(I_{1}+I_{2}\right)
$$

as long as this is a small quantity. The observed oscillations in $R_{x x}$ have amplitude of approximately $2 \Omega$ indicating a suppression factor of $Q \simeq 0.01$. Higher-order interference terms will be suppressed by higher powers of $P$ and $Q$, so combining these we find that the amplitude of double pass interference oscillations is expected to be roughly 0.0005 times that of the lowest order oscillation amplitude. Hence, the $\Delta A=2 \Phi_{0} / B$ oscillations, for which the amplitudes are of the same order of magnitude as that of the $\Delta A=4 \Phi_{0} / B$ oscillations, should be attributed almost entirely to the tunneling of $e / 2$ edge quasiparticles. We emphasize that the $\Delta A$ $=2 \Phi_{0} / B$ oscillations (from both sources) have an amplitude that is independent of $n_{q}$ (unlike the $\Delta A=4 \Phi_{0} / B$ oscillations), but pick up phase shifts when $n_{q}$ changes.

These two sources of $\Delta A=2 \Phi_{0} / B$ oscillations were not discussed in Refs. 32 and 33 because it was assumed neither would have significant contributions to the tunneling current. For the double pass interference of $e / 4$ quasiparticles, this appears to be a valid assumption, since higher-order tunneling processes are suppressed in the weak-backscattering regime. On the other hand, for interference of $e / 2$ quasiparticles this assumption was based on such quasiparticles having less relevant tunneling operators than the $e / 4$ quasiparticles. We will see in the following that there are several ways in which this line of reasoning can break down and permit the $e / 2$ quasiparticles to have a contribution to the tunneling current oscillations that is comparable to that of the $e / 4$ quasiparticles.

Combining these results, we see that tunneling of both non-Abelian $e / 4$ quasiparticles and Abelian $e / 2$ quasiparticles at the point contacts of the interferometer would produce a combined backscattered current with regions of type I exhibiting a sum of both $\Delta A=4 \Phi_{0} / B$ and $\Delta A=2 \Phi_{0} / B$ oscillations, when $n_{q}$ is even, and regions of type II, exhibiting only $\Delta A=2 \Phi_{0} / B$ oscillations, when $n_{q}$ is odd. We also note that the bulk-edge coupling that occurs as a bulk $e / 4$ quasiparticle approaches the edge gives the regions near transitions between type I and II oscillations the most potential for exhibiting nonlinear and/or noisy behavior. The behavior of the interference current in the weak to strong coupling crossover as the quasiparticle approaches the edge was recently studied in Refs. 51 and 52, which found results not entirely inconsistent with the experimental data.

In order for interference to be observed, it is necessary that the current-carrying excitations remain phase coherent. Even if we neglect (irrelevant) interactions between the edge modes, coupling to localized excitations in the bulk, and phonons, there will still be thermal smearing of the interference pattern. Consequently, as shown in Ref. 53 (see also Refs. 54 and 55), the amplitude of interference oscillation for double point-contact interferometers will be exponentially suppressed in temperature and in the average length $L$ between point contacts along each edge 
TABLE I. Estimated coherence lengths $L_{\phi}$ at $T=25 \mathrm{mK}$ and coherence temperatures $T^{*}$ for $L=1 \mu \mathrm{m}$ for the (relevant) $e / 4$ quasiparticles of the candidate $\nu=5 / 2$ states and the $e / 2$ Laughlin-type quasiparticle for all these states. We use the velocity estimates $v_{c}$ $\approx 5 \times 10^{4} \mathrm{~m} / \mathrm{s}$ and $v_{n} \approx 4 \times 10^{3} \mathrm{~m} / \mathrm{s}$ from numerical studies (Ref. 56), while the temperature $T=25 \mathrm{mK}$ and path length $L=1 \mu \mathrm{m}$ are characteristic of the experiments of Willett et al. (Refs. 42-44).

\begin{tabular}{lcccc|c}
\hline \hline$e / 4$ & $\mathrm{MR}$ & $\overline{\mathrm{Pf}} / \mathrm{SU}(2)_{2}$ & $K=8$ & $(3,3,1)$ & $e / 2$ \\
\hline$L_{\phi}$ in $\mu \mathrm{m}$ & 1.4 & 0.5 & 19 & 0.7 & 4.8 \\
$T^{*}$ in $\mathrm{mK}$ & 36 & 13 & 484 & 19 & 121 \\
\hline \hline
\end{tabular}

$$
I_{12}^{(\mathrm{qp})} \propto e^{-T / T^{*}(L)}=e^{-L / L_{\phi}(T)},
$$

where the coherence length $L_{\phi}(T)$ and temperature $T^{*}(L)$ of edge excitations are given by

$$
\begin{aligned}
& L_{\phi}(T)=\frac{1}{2 \pi T}\left(\frac{g_{c}}{v_{c}}+\frac{g_{n}}{v_{n}}\right)^{-1}, \\
& T^{*}(L)=\frac{1}{2 \pi L}\left(\frac{g_{c}}{v_{c}}+\frac{g_{n}}{v_{n}}\right)^{-1} .
\end{aligned}
$$

We can use these expressions, together with estimates of the charge and neutral edge mode velocities $\left(v_{c} \approx 5 \times 10^{4} \mathrm{~m} / \mathrm{s}\right.$ and $v_{n} \approx 4 \times 10^{3} \mathrm{~m} / \mathrm{s}$ ) from numerical studies ${ }^{56}$ of $\nu=5 / 2$ with pure Coulomb interactions on a $\operatorname{disk}^{86}$ (the charged and neutral scaling exponents $g_{c}$ and $g_{n}$ are given in Table II), to estimate coherence lengths and temperatures for the charge $e / 4$ and $e / 2$ excitations in the various candidate states (the states are all the same, as far as the charge $e / 2$ quasiparticle is concerned). In Table I, we give estimates of coherence lengths at $T=25 \mathrm{mK}$ and coherence temperatures for $L$ $=1 \mu \mathrm{m}$. The temperature $T=25 \mathrm{mK}$ is the lowest temperature at which the experiments of Refs. 42-44 were carried out, and $L=1 \mu \mathrm{m}$ is the approximate interference path length on each side of the interferometry area determined in Refs. $42-44$ to be $A \approx 0.2 \mu \mathrm{m}^{2}$. We note that the observation of only type II oscillations at higher temperatures in Refs. 42-44 also excludes double pass interference of $e / 4$ quasiparticles as the explanation for $\Delta A=2 \Phi_{0} / B$ oscillations, whereas it fits very nicely with the $e / 2$ quasiparticle tunneling explanation.

\section{B. Coulomb blockade}

If the region between the two point contacts in the experiment of Willett et al. $^{42-44}$ was nearly an isolated puddle, the Coulomb charging energy of the puddle would dominate the behavior of the device. This might occur if the gates pinched off the point contact too strongly. Due to its isolation, the puddle must contain an integer number of electrons. The electron number can change when the gate voltage is increased by enough to allow one additional electron into the puddle. At this point, there is a peak in the longitudinal conductance (which is also a peak in the longitudinal resistance, since $R_{L} \ll R_{H}$ ) since it is only at this point (or within $k_{B} T$ of it) that the charge on the puddle can fluctuate. The maxima
TABLE II. Relevant quasiparticle excitations of model FQH states at $\nu=5 / 2$. Here we list their values of charge $e^{*}$; whether they are non-Abelian; their topological twist factor $\theta$; and their charge and neutral scaling exponents $g_{c}, g_{n}$, and $g$. The MR, $\overline{\mathrm{Pf}}$, and $\mathrm{SU}(2)_{2}$ NAF states are non-Abelian, while the $K=8$ (strong pairing) and $(3,3,1)$ states are Abelian. All of these have Abelian $e / 2$ Laughlin-type quasiparticles.

\begin{tabular}{lllcccc}
\hline \hline$\nu=\frac{5}{2}$ & $e^{*}$ & $\mathrm{n}-\mathrm{A} ?$ & $\theta$ & $g_{c}$ & $g_{n}$ & $g$ \\
\hline $\mathrm{MR}:$ & $e / 4$ & Yes & $e^{i \pi / 4}$ & $1 / 8$ & $1 / 8$ & $1 / 4$ \\
& $e / 2$ & No & $e^{i \pi / 2}$ & $1 / 2$ & 0 & $1 / 2$ \\
\hline$\overline{\mathrm{Pf}}:$ & $e / 4$ & Yes & $e^{-i \pi / 4}$ & $1 / 8$ & $3 / 8$ & $1 / 2$ \\
& $e / 2$ & No & $e^{i \pi / 2}$ & $1 / 2$ & 0 & $1 / 2$ \\
\hline $\mathrm{SU}(2)_{2}:$ & $e / 4$ & Yes & $e^{i \pi / 2}$ & $1 / 8$ & $3 / 8$ & $1 / 2$ \\
& $e / 2$ & No & $e^{i \pi / 2}$ & $1 / 2$ & 0 & $1 / 2$ \\
\hline$K=8:$ & $e / 4$ & No & $e^{i \pi / 8}$ & $1 / 8$ & 0 & $1 / 8$ \\
& $e / 2$ & No & $e^{i \pi / 2}$ & $1 / 2$ & 0 & $1 / 2$ \\
\hline$(3,3,1):$ & $e / 4$ & No & $e^{i 3 \pi / 8}$ & $1 / 8$ & $1 / 4$ & $3 / 8$ \\
& $e / 2$ & No & $e^{i \pi / 2}$ & $1 / 2$ & 0 & $1 / 2$ \\
\hline \hline
\end{tabular}

of the oscillations seen in Willett et al.'s experiment ${ }^{42-44}$ would be these peaks. If the density in the puddle is fixed, then the spacing between peaks as a function of area is naively just the additional area required to allow one more electron into the puddle:

$$
\Delta A=\frac{e}{\rho_{0}},
$$

where $\rho_{0}$ is the charge density inside the dot. However, in the case of a paired state, one would expect that it is easier to add an electron when the electron number is odd than when it is even since, in the latter case, an unpaired fermionic excitation is necessarily created. So one would expect that, instead of evenly spaced peaks, the interval between an odd peak and the next even peak would be smaller than the interval between an even peak and the next odd peak because $V_{s}$ must also supply the energy needed to create an unpaired fermionic excitation. Consequently, the peak spacing would alternate between ${ }^{57}$

$$
\Delta A_{ \pm}=\frac{e}{\rho_{0}}\left(1 \pm \frac{v_{n}}{2 v_{c}}\right) .
$$

As a result of this "bunching" effect, the periodicity would be the interval between two successive even peaks, i.e., twice what one might ordinarily expect. But when there is an odd number of charge $e / 4$ quasiparticles in the $\mathrm{MR}, \overline{\mathrm{Pf}}$, or $\mathrm{SU}(2)_{2}$ states, the minimum energy to create a fermionic excitation is zero. Thus, there is no bunching effect in this case and the period is not doubled. ${ }^{32}$

In the case of the $(3,3,1)$ state, bunching generically occurs with either an even or odd number of quasiparticles in the puddle. However, when $n_{q}$ is odd, the bunching depends on the strength of the violation of $S_{z}$ conservation (where $S_{z}$ is the $z$ component of the spin or, if one contemplates a 
bilayer version of this experiment, the layer pseudospin) so $v_{n} / 2 v_{c}$ in Eq. (10) is replaced by a different constant dependent on this violation. This is because the neutral sector of the edge theory of the $(3,3,1)$ state is a pair of Majorana fermions or, equivalently, a Dirac fermion (which can be bosonized, leading to the standard $K$-matrix description). If $S_{z}$ is conserved (or only weakly nonconserved), both Majorana fermions will have edge zero modes for odd $n_{q}$ and the bunching will disappear. In this case, the bunching pattern would look just like that predicted for the MR, $\mathrm{Pf}$, and $\mathrm{SU}(2)_{2}$ states. However, if $S_{z}$ is more than weakly nonconserved, then the two Majorana fermions zero modes will mix and split, leaving no zero modes. Consequently, when $S_{z}$ is not conserved, there will be bunching even when there is an odd number of quasiparticles. Thus, switching between bunching and nonbunching regions in Coulomb blockade at $\nu=5 / 2$ is not necessarily an indication of a non-Abelian state. Furthermore, if $v_{n} \ll v_{c}$, bunching will never be seen in any state. More generally, the switching between different bunching patterns in Coulomb blockade described in Refs. 32 and 57 for non-Abelian states may similarly be mimicked by corresponding Abelian states (for more details, see Ref. 58 where explicit examples are given for all the most physically relevant non-Abelian states).

The strongly paired $K=8$ state $^{28}$ always exhibits bunching, now with $v_{n} / 2 v_{c}$ in Eq. (10) replaced by a constant dependent upon the finite energy cost of having an unpaired electron. If this energy cost is small, it may not appear bunched. On the other hand, if it is large enough, it will be maximally bunched with $\Delta A=2 e / \rho_{0}$ corresponding to tunneling electron pairs.

\section{Nonlinear area vs $V_{s}$ dependence}

The assumptions that $\Delta A=c \Delta V_{s}$ with only a single value of $c$ across a range of filling factors and a range of $V_{s}$ values are important for the two previous interpretations of this experiment. It is not clear that $d A / d V_{s}$ should be constant across an appreciable range of $V_{s}$ values because the density is not constant across the device. In fact, we expect $V_{s}$ to vary linearly with total charge in the central puddle. So long as the electron density is essentially fixed, apart from a small number of quasiparticles, $V_{s}$ will vary linearly with $A$. However, if there are high-density and low-density regions, then we will have $\Delta A=c \Delta V_{s}$ in some regions and $\Delta A=c^{\prime} \Delta V_{s}$ in the others, with $c \neq c^{\prime}$. (This could lead, for instance, to $\nu$ $=7 / 3$ puddles within the $\nu=5 / 2$ droplet.) This would, in turn, lead to two different regions with different oscillation periods. However, it is difficult to see why one period would be twice the other or why there would be two periods only at $\nu=5 / 2$ and not at $\nu=5 / 3,2$, and $7 / 3$.

One other possibility, which also depends on spatial inhomogeneity although still assuming a linear $A$ vs $V_{s}$, is that there are regions in the sample in which the $K=8$ Abelian state occurs. The rest of the state is assumed to be nonAbelian, i.e., either the MR or $\overline{\mathrm{Pf}}$ state. Then, when a $K=8$ region is at the edge of the system, varying $V_{s}$ does not change the area enclosed by the edge of the non-Abelian part of the system, which would lead to $e / 4$ oscillations. It does cause the total area to vary, but this only causes $e / 2$ oscillations since these oscillations can move coherently along both $K=8$ Abelian and non-Abelian edges. Thus, the two regions correspond to when the edge of the system near the side gate is a $K=8$ region or a non-Abelian region.

\section{CRITICAL ANALYSIS}

\section{A. Non-Abelian interference}

The data of Refs. 42-44 are broadly consistent with the hypothesis that the device is functioning as a quantum Hall edge state interferometer. As the temperature is raised, the putative $e / 4$ oscillations, which are observed at $30 \mathrm{mK}$, disappear while the $e / 2$ oscillations persist even at $150 \mathrm{mK}$. This was anticipated in Ref. 56, where it was noted that the coherence length will be substantially longer for $e / 2$ quasiparticles than for $e / 4$ quasiparticles since the former do not involve the slow neutral edge modes. Thus, any of the proposed $\nu=5 / 2$ states (apart from the strong-pairing state) would be broadly consistent with the $e / 4$ oscillations seen in Refs. 42-44. However, there is no simple explanation of their absence in the type II regions in the $(3,3,1)$ state, while the $\mathrm{MR}, \overline{\mathrm{Pf}}$, and $\mathrm{SU}(2)_{2}$ states all provide a simple explanation, as described in the previous section. One, however, needs a more careful and detailed study of the temperature and voltage behavior before a favored candidate non-Abelian state can be identified.

Perhaps the most serious challenge to the non-Abelian interferometer hypothesis is that the same amplitude of $e / 2$ oscillations should always be present (i.e., in type I and II regions), while $e / 4$ oscillations should only be observed when the quasiparticle number contained within the interferometry region is even. This appears to be the case in Figs. 3 and S2b of Ref. 43 and Fig. 2a of Ref. 44, and to a lesser extent in Figs. 4b and S2a of Ref. 43 and Fig. 2c of Ref. 44. However, this appears not to be the case in Fig. 4a of Ref. 43 and Fig. $2 b$ of Ref. 44 . It is possible to generate some accidental destructive interference between the oscillations due to tunneling of $e / 2$ quasiparticles given in Eq. (2) and that of double pass interference of $e / 4$ quasiparticles given in $\mathrm{Eq}$. (3), since the relative phase of these terms is not fixed. This could potentially result in the appearance and disappearance of $e / 2$ oscillations, however, as previously mentioned, the magnitude of oscillations in Eq. (2) is so strongly suppressed in the experiments of Refs. 42-44 that it could not explain such behavior there.

As we describe in Appendix B, a simple model of quasiparticle tunneling predicts that the amplitude for $e / 4$ quasiparticle backscattering, $\Gamma_{e / 4}$, is much larger than the amplitude for $e / 2$ quasiparticle backscattering, $\Gamma_{e / 2}$. However, the magnitude of $e / 2$ oscillations in the type II regions is comparable to the magnitude of $e / 4$ oscillations in the type I regions. It may be that $\left|\Gamma_{e / 2}\right|$ is "accidentally" large, e.g., due to the presence of a tunneling resonance for $e / 2$ quasiparticles at the point contact. (For example, such a resonance might occur if there is an impurity or a region of different filling in the point contact acting like a dot or antidot through which resonant tunneling favors the $e / 2$ charge.) Alternatively, as a result of the shorter coherence length for $e / 4$ 
excitations, the corresponding oscillations are more strongly suppressed. This would require a coincidence-that thermal smearing of $e / 4$ excitations compensates for the smallness of the ratio $\Gamma_{e / 2} / \Gamma_{e / 4}$. However, this could be tested by going to lower temperatures to reduce the suppression, and by increasing the separation between the point contacts to increase the suppression. At any rate, given that $e / 2$ oscillations are observed in the type II regions, it would be a problem for the non-Abelian interferometer picture if they are not generically seen in the type I regions.

However, it is worth noting in this context that the presence of charge $e / 2$ quasiparticle tunneling is not manifest in the point-contact experiments of Refs. 22 and 23. In the former, the shot noise appears to indicate that only charge $e / 4$ quasiparticles tunnel at the point contact (although there is sufficient scatter in the data that one might argue that there could be a component due to $e / 2$ quasiparticle, the scatter does not seem to be asymmetric in the direction of charges larger than $e / 4$ as one might have expected). In the latter experiment, the best fit to the data is actually $e^{*} / e=0.17$, so including any $e / 2$ tunneling leads to a worse fit to the data. ${ }^{23}$ Thus, the appearance and strength of $e / 2$ quasiparticle tunneling remain a mystery in several different experiments.

\section{B. Coulomb blockade}

A conventional Coulomb blockade picture seems inappropriate since $I_{b} \lesssim 0.1 I_{\text {total }}$ indicates that the system is in the weak backscattering limit. It is also unlikely that Coulomb blockade could lead to two distinguishable periods since, for $v_{n} / 2 v_{c}$ small (as we expect it to be), the bunching will be difficult to resolve. Numerical calculations of the edge velocities $^{56}$ give $v_{n} \simeq 0.1 v_{c}$ confirming this expectation. On the other hand, we note that Coulomb blockade is capable of producing peaks that alternate between the $e / 4$ and $e / 2$ periodicities, with no $e / 2$ background in the $e / 4$ region. Thus, if the two prior points against it were somehow incorrect, Coulomb blockade could provide a consistent explanation of the periodicity issue.

Furthermore, Coulomb blockade could be easily ruled out by measuring its temperature dependence and its dependence on asymmetry between the tunneling amplitudes at the two point contacts. In particular, the Coulomb blockade peak widths are expected to scale linearly with temperature. ${ }^{59}$ However, a more general view of Coulomb blockade has emerged $^{38}$ (see also Refs. 60 and 61) according to which Coulomb blockade (CB) can be distinguished from AB interference by inter alia the dependence of $\Delta V_{s}$ on $B$ (it should be inversely proportional for $\mathrm{AB}$ and independent for $\mathrm{CB}$ ). This more general view of Coulomb blockade is probably better described as "Coulomb dominated" since it corresponds to a regime in which the charging energy of the puddle between the point contacts is the dominant energy scale. It does not rule out a simple interpretation of the backscattered current according to Eq. (1).

At any rate, by this criterion as well, the data appear to be more consistent with $\mathrm{AB}$ interference since $\left(\Delta V_{s}\right)_{5 / 3} B_{5 / 3}$ $\approx\left(\Delta V_{s}\right)_{7 / 3} B_{7 / 3} \approx 3\left(\Delta V_{s}\right)_{2} B_{2}$. However, it is worth keeping in mind that we do not know precisely how the area of the droplet changes with $V_{s}$ or with $B$ (the $B$ dependence further distinguishes Aharonov-Bohm from Coulomb blockade because $A$ should be independent of $B$ in the former case, but not in the latter case); knowing this would enable us to cement an interpretation of the experiment.

\section{Nonlinear area vs $V_{s}$ dependence}

As previously mentioned, one might question the validity of the assumption that $\Delta A=c \Delta V_{s}$ holds with the same value of $c$ across a range of filling fractions. However, the assumption that $c$ is independent of the filling fraction for nearby filling fractions is, in fact, reasonable. $V_{s}$ is several volts and the oscillation periods are $\sim 10 \mathrm{mV}$ which are much higher energy scales than the weak energy gaps and correlation effects associated with the $\nu=5 / 3,7 / 3$, and 5/2 quantum Hall states. Thus, the details of these quantum Hall states are probably unimportant and $d A / d V_{s}$ is probably determined by the electric potential due to the donor impurities and the electron density, which are not varying significantly. However, when there are filled Landau levels beneath the quantum Hall state of interest, their edges can screen the side-gate voltage presumably weakening the dependence of $A$ on $V_{s}$ (since $A$ is the area of the droplet of the fractional state in the partially filled Landau level). In particular, we would expect $\Delta A=c_{1} \Delta V_{s}$ at $\nu=1 / 3$ but $\Delta A=c_{2} \Delta V_{s}$ at $\nu=7 / 3$, with $c_{1}$ $>c_{2}$. However, by the same reasoning, we expect that the relationship between $A$ and $V_{s}$ will be the same for $\nu=5 / 3$, $7 / 3$, and $5 / 2$ (if the $\nu=5 / 3$ edge is two filled Landau levels with a backward propagating $\nu=1 / 3$ edge mode).

\section{ADDITIONAL PROPOSALS AND NONTRIVIAL CHECKS}

As beautiful as the non-Abelian anyon explanation of the results of Ref. 42-44 may be, it is clear from the preceding analysis that there are some significant gaps which need to be closed through further measurements.

\section{A. Temperature and voltage scaling behavior}

If it is indeed the case that $R_{L}$ is due to the weak backscattering of $e / 4$ quasiparticles at the constrictions, then both the nonoscillatory and oscillatory parts of the current should have nontrivial temperature and voltage dependence. Modeling the edge in the simplest way (i.e., fully equilibrated neutral modes and no edge reconstruction) using the "natural" conformal field theory inherited from the bulk, one can perform a more detailed analysis of the tunneling edge current, ${ }^{24,25,50,62}$ along the lines of that carried out in Refs. 29 and 63 for Abelian states.

The nonoscillatory part of the backscattered current- the sum of the contributions from each point contact independently - will behave as the power laws:

$$
I_{b}^{(\mathrm{qp})} \propto\left\{\begin{array}{ll}
T^{2 g-2} V & \text { for small } e V \ll k_{B} T \\
V^{2 g-1} & \text { for small } e V \gg k_{B} T
\end{array},\right.
$$

where $g=g_{c}+g_{n}$ is the tunneling exponent combining charge and neutral (Abelian and non-Abelian) sectors of the quasi- 
particles' tunneling operator. The tunneling operator is relevant for $g<1$, and quasiparticles with smaller $g$ are more relevant, and are thus expected to dominate the tunneling current in the weak backscattering limit.

From Table II, we see that the $e / 4$ backscattering operator is a relevant perturbation of the edge effective theory for all of the candidate states. Thus, the effective tunneling amplitude(s) will decrease as the temperature is raised, as $T^{-3 / 2}$, $T^{-5 / 4}$, or $T^{-1}$ in the $\mathrm{MR},(3,3,1)$, or $\overline{\mathrm{Pf}}$ and $\mathrm{SU}(2)_{2}$ states, respectively. Charge $e / 2$ backscattering is also relevant in all of the candidate states. Because $e / 2$ excitations have $g$ $=1 / 2$ and are entirely in the charge sector, their lowest order, single point-contact tunneling current contribution is the same in all of the candidate states. Using the methods of Ref. 63 to explicitly compute its value, we find

$$
I_{b}^{(e / 2)}=\frac{e}{2}\left|\Gamma_{e / 2}\right|^{2} \frac{2 \pi}{v_{c}} \tanh \left(\frac{e V}{4 T}\right) .
$$

Thus, in the linear-response regime, the effective tunneling amplitude for $e / 2$ backscattering decreases as $T^{-1}$. For the MR and Abelian states, charge $e / 4$ backscattering is more relevant than charge $e / 2$ backscattering, so it is expected to dominate at lower temperatures. For the $\overline{\mathrm{Pf}}$ and $\mathrm{SU}(2)_{2}$ states, $e / 4$ and $e / 2$ backscattering are equally relevant (with $g=1 / 2$ ). As previously mentioned in Sec. I, the single pointcontact experiment of Ref. 23 found voltage and temperature dependence of the tunneling current to be most consistent with the $\overline{\mathrm{Pf}}$ and $\mathrm{SU}(2)_{2}$ states providing a point of reference with which to compare.

Turning now to the oscillatory current, we note that for $e V \ll v_{n} / 2 L$, where $2 L$ is the interference path length, it has the same voltage dependence as the nonoscillatory current. For larger voltages, it becomes apparent that there are oscillations with a period $\sim 4 \pi v_{n} / L$, but these are much larger voltages than are probed in the experiments of Refs. 42-44. We note that these oscillations can be turned around and interpreted as oscillations as a function of $L$, which changes when the interferometry area is changed (i.e., when the sidegate voltage is changed). However, these oscillations have periodicity $4 \pi v / e^{*} V$, where $v$ represents several characteristic velocities, which are all dominated by the slowest edge mode velocity (which is expected to be $v_{n}$ ). Since $V$ $\simeq 10^{-8} \mathrm{~V}$, these will only give rise to envelopes with periods much longer than that of the oscillations observed in the experiment.

The temperature dependence of the oscillatory current includes a power-law prefactor of the form in Eq. (11) in addition to the exponential suppression $e^{-T / T^{*}(L)}=e^{-L / L_{\phi}(T)}$ which we discussed earlier. Thus, the relative suppression of the $e / 4$ contribution, compared to the $e / 2$ contribution, must be due entirely to the shorter coherence length in the $\overline{\mathrm{Pf}}$ case but could be due to a combination of effects in the MR or $(3,3,1)$ case.

To make the case for interference stronger, it would be helpful to disentangle the effects of the temperature dependence of the coherence length from the temperature dependence of the effective tunneling amplitude. One way to do this would be to carefully study the bias voltage dependence of the current backscattered by the interferometer of Refs. 42-44 at some fixed $V_{s}$ in the low- $T$ limit. If the behavior is similar to that observed in Ref. 23 (and, especially, if it is the behavior expected for one of the possible $\nu=5 / 2$ states), then this is a strong indication that $R_{L}$ is due to the weak backscattering of charge $e / 4$ quasiparticles. Another useful way to do this would be to turn on the point contacts one at a time and study their tunneling behavior individually. This would help determine which state occurs in the point-contact region; it is important that it is at the same filling fraction as the rest of the bulk. Furthermore, it would allow one to determine the relative tunneling amplitudes of $e / 4$ and $e / 2$ quasiparticles and confirm that the experiment is not in the CB regime.

\section{B. Fourier analysis}

It is important to verify that the oscillation periodicities behave as expected. In addition to confirming the dominance of $\Delta A=4 \Phi_{0} / B$ and $\Delta A=2 \Phi_{0} / B$ oscillations in the Fourier spectrum, one should examine the spectrum in the different regions more carefully. Specifically, by using windowing techniques in the Fourier analysis of the data, one should check that the type I regions have both $\Delta A=4 \Phi_{0} / B$ and $\Delta A=2 \Phi_{0} / B$ oscillations, that the type II regions have only $\Delta A=2 \Phi_{0} / B$ oscillations, and that the amplitude of $\Delta A$ $=2 \Phi_{0} / B$ oscillations are roughly the same in the type I and II regions. It is also useful to know the relative oscillations amplitudes of the two frequencies in the type I regions.

\section{Significance of $\pi / 2$ and $\pi$ phase shifts}

Another aspect of the data worth examining more closely is the phase shifts observed in the oscillations of a given type. While an $e / 4$ bulk quasiparticle entering or exiting the interference loop causes a switch between type I and II oscillations, there could also be $e / 2$ quasiparticles in the bulk that enter or exit the interference loop when the area is changed (or pairs of $e / 4$ quasiparticles that enter or exit nearly simultaneously), which would not switch the oscillations between type I and II. From Eq. (2) we see that this would cause a shift of phase $\pi$ in the $e / 2$ oscillations, while from Eq. (1) we see that this would cause a shift of phase $\pi / 2$ in the $e / 4$ oscillations. To more firmly establish the origin of these phase shifts, as well as how reproducible are the fine details of the observed oscillations, one should immediately backtrack on the side-gate voltage within an oscillation region of fixed type to see whether the oscillations (including locations of phase shifts) are nearly exactly reproduced. Furthermore, we recall that if one is in a type I region and an $e / 4$ bulk quasiparticle exits and then re-enters the interference loop, the value of $n_{\psi}$ in Eq. (1) will be randomized with equal probability. Hence, one should also increase the side-gate voltage (decreasing the interferometry area) until one transitions from a type I region into a type II region, and then soon afterward backtrack on the side-gate voltage to return to the type I region. When this is done repeatedly, if the e/4 oscillations in the type I region are found to be shifted by a phase of $\pi$ half the time, it would be a direct observation of non-Abelian statistics. Doing this backtrack- 
ing immediately would provide a more convincing observation of the $\pi$ phase shift due to the non-Abelian statistics than backtracking after a complete run, which leaves plenty of time (1 day) for small changes to occur that may give nonuniversal phase shifts. Furthermore, it is more convenient to perform a statistical experiment for which each sampling takes $1 \mathrm{~h}$ rather than 1 day to complete.

\section{Area of the interferometry region}

It is important to establish that the periodicities with $V_{s}$ obtained in the experiment correspond directly to periodicities with $A$. One way to attempt to do this would be to vary both $V_{s}$ and $B$ at $\nu=2$ and to use the periodicity in $B$ to determine the area for several different values of $V_{s}$. One could, in this way, check that the assumed constant $c$ in $\Delta A=c \Delta V_{s}$ is really constant. Such a measurement would also determine whether the oscillation pattern corresponds to $\mathrm{AB}$ interference or Coulomb blockade, as in Ref. 38. One could also check that the oscillations are due to $\mathrm{AB}$ interference by turning down or off one of the point contacts (and then the other) and repeating the experiment, which should cause the oscillations to disappear. This will further exclude resonances at a single point contact as the source of oscillations and will give a better value of the tunneling amplitude for a single point contact.

A more ambitious approach to measuring the area within the interference loop, which could simultaneously tackle the even more fundamental problem of determining directly whether the type I and II regions correspond to even/odd quasiparticle numbers, would be to image the twodimensional electron gas (2DEG) in the interferometer using a scanning single electron transistor (SET), as in Ref. 64. In this earlier experiment, a scanning SET was used to image the compressibility of the electron liquid at $\nu=1,1 / 3$, and $2 / 3$. By measuring the compressibility, it should be possible to determine where the edge of the Hall fluid is in the droplet and, hence, the area of the interferometry region. It should also be possible to find the localized states near the Fermi energy where $e / 4$ quasiparticles could be trapped. By imaging the charge $e / 4$ quasiparticles, one might even be able to see these localized quasiparticles enter or leave the interference loop as $V_{s}$ is varied, and hence allow the most direct verification of the non-Abelian interferometer interpretation. Similarly, the possibility that the existence of two periodicities signals different regions with different electron densities and, therefore, two different possible relations $\Delta A=c \Delta V_{s}$ and $\Delta A=c^{\prime} \Delta V_{s}$ could be ruled in or out through a more detailed knowledge of the electron density in the sample.

\section{E. Tracking bulk quasiparticles}

We note, as a consistency check on the data of Refs. 43 and 44 , that one can use the area periodicity of the AB oscillations to estimate the density of bulk $e / 4$ quasiparticles by attributing each observed switching between type I and II regions to an $e / 4$ quasiparticle entering or leaving the interferometry region. In this way, we estimate the density to be $\rho_{e / 4} \approx 50 \mu \mathrm{m}^{-2}$. This translates to $\sim 10$ charge $e / 4$ quasiparticles in the interference loop. We can also estimate the num- ber of $e / 2$ quasiparticles in the bulk, though perhaps less reliably, by attributing phase disruptions observed within an oscillation type region to an $e / 2$ quasiparticle crossing the interferometry region. Using this to similarly estimate the density of bulk $e / 2$ quasiparticles gives $\rho_{e / 2} \approx 50 \mu \mathrm{m}^{-2}$ or roughly the same number as charge $e / 4$ quasiparticles in the interference loop. Depending on how seriously one takes the $e / 2$ contribution, this gives approximately $0.5 \%-1.5 \%$ depletion of the electron density in the bulk, which is the reported density variation in the device. ${ }^{42-44}$ With a scanning SET setup, it may be possible to find the $\sim 10$ charge $e / 4$ quasiparticles that are necessary for the non-Abelian interferometer interpretation and observe them entering or exiting the interference loop. Localized $e / 2$ quasiparticles or, equivalently, closely spaced pairs of $e / 4$ quasiparticles should also be observable.

\section{F. Multiple plungers}

A more crude but also more easily implementable way to further strengthen the correlation between which oscillation type is observed and the localization of excitations in the bulk is to independently vary two or more plunger gates of the interferometer. By refining the ability to control how the interferometry area is changed beyond a single plunger variable, the changes between oscillation types can be more strongly associated with a particular area. If a region of one plunger's gate voltage exhibits type I oscillations, but then, after changing a separate plunger's position, the same voltage range in the first plunger exhibits type II oscillations, this would demonstrate that a particular oscillation type is not associated with that particular voltage range of the first plunger, but rather that an ability to change between oscillation types is associated with a localized quantity in the area added or removed by the second plunger. This would greatly strengthen the evidence for non-Abelian braiding statistics.

\section{G. Other second Landau level states}

A double point-contact interferometer may also be used to test whether the quantum Hall states at $\nu=7 / 3,12 / 5,8 / 3$, and $14 / 5$ are non-Abelian. These filling fractions all have compelling Abelian alternatives which almost certainly occur at their corresponding lowest Landau level counterparts. While numerical studies strongly support the MR and $\overline{\mathrm{Pf}}$ states at $\nu=5 / 2$ (Refs. 56 and 65-72) and the (particle-hole conjugate) Laughlin state at $\nu=14 / 5,{ }^{73,74}$ they are far less conclusive for $\nu=7 / 3,12 / 5$, and $8 / 3,{ }^{74-77}$ where several candidates seem plausible including ones that are non-Abelian. It is clearly important to also test these FQH states experimentally. The details of the plausible candidates' experimental signatures are discussed in Appendix A. The signatures of non-Abelian statistics in these states will again be dramatic, though not quite as much as for the $\mathrm{MR}, \overrightarrow{\mathrm{Pf}}$, or $\mathrm{SU}(2)_{2} \mathrm{NAF}$ states.

\section{DISCUSSION}

We close this discussion by assuming, for a moment, that the experiments of Refs. 42-44 are, in fact, performing in- 
terferometry on the $\nu=5 / 2$ state of the sort envisioned in Refs. 29-33 and are detecting non-Abelian quasiparticles. What forecast would these results give for topological quantum computation? ${ }^{14-21}$ Certainly, it would be encouraging that a non-Abelian topological state, the sine qua non for topological quantum computing, would be found. One potential source of concern is the appearance of some seemingly unpredictable phase disruptions, which would make it difficult to distinguish the two states of a topological qubit, which differ by a $\pi$ phase shift in their ( $e / 4$ oscillation) interference patterns. However, if further investigation shows that they are $\pi / 2$ phase shifts (in the type I regions), then they may be attributable to $e / 2$ quasiparticles or pairs of $e / 4$ quasiparticles simultaneously entering or exiting the interference loop, and it would be a manageable problem. On the other hand, if they turn out to be $\pi$ phase shifts in the $e / 4$ oscillations, then they could be attributed to either tunneling of a $e / 4$ quasiparticle from one edge to the other between bulk quasiparticles within the interferometry region or tunneling of an electrically neutral fermion between the edge and a bulk quasiparticle within the interferometry region. Either of these $\pi$ phase shifting processes would cause errors in topological qubits, so if we attribute all the observed phase disruptions to such processes, this gives a crude estimate of about $1 \mathrm{~h}$ for the time scale for such errors or a contribution to the topological qubit error rate of $\Gamma / \Delta \lesssim 10^{-13}$. To better determine the rate of such errors, one could simply tune the voltage to a local $e / 4$ oscillation maximum/minimum of the tunneling current in a type I region and time average duration it takes for the current to jump to a lower/higher value (i.e., experiencing a $\pi$ phase shift to a minimum/maximum of the current). If the phase disruptions are neither $\pi / 2$ nor $\pi$ phase shifts, then they would be a serious concern, as they would have no obvious explanation. Finally, the apparent stability of the type I and type II regions implies that thermally activated charge $e / 4$ quasiparticles do not move in and out of the interferometry region over the time scales of this experiment. Indeed, these regions are stable on a time scale of a week, which would imply a topological qubit error rate from mobile bulk quasiparticles of $\Gamma / \Delta \leq 10^{-15} \cdot{ }^{87}$

\section{ACKNOWLEDGMENTS}

We would like to thank R. Willett for many important discussions and access to unpublished work, M. Heiblum for a discussion of unpublished work, and S. Das Sarma, M. Freedman, and Y. Gefen for helpful comments. W.B., K.S., and J.K.S. would like to acknowledge the support and hospitality of Microsoft Station Q. P.B., C.N., and K.S. would like to acknowledge the hospitality of the Aspen Center for Physics. C.N. and K.S. were supported in part by the DARPA-QuEST program. K.S. was supported in part by the NSF under Grants No. DMR-0748925 and No. PHY05-51164, and would like to acknowledge the hospitality of the KITP. J.K.S. was supported in part by the Science Foundation of Ireland Principal Investigator Grant No. 08/ IN.1/I1961.

\section{APPENDIX A: OTHER SECOND LANDAU LEVEL STATES}

In this section, we consider the other observed $\mathrm{FQH}$ states in the second Landau level, ${ }^{78,79}$ i.e., $\nu=7 / 3,12 / 5,8 / 3$, and
14/5. We provide the data of their prominent candidate descriptions that will be useful for interpreting tunneling and interference experiments, similar to earlier in this paper.

As described earlier, the interference term of the tunneling current combines the following: (1) the AB effect, (2) the braiding statistics with encircled quasiparticles, and (3) the edge physics. The $\mathrm{AB}$ effect simply contributes a phase $e^{i e^{*} \Phi / \hbar c}=e^{i 2 \pi e^{*} \Phi / e \Phi_{0}}$ when the quasiparticle of charge $e^{*}$ encircles flux $\Phi$. The physics describing propagation of excitations on the edge gives rise to a temperature, bias voltage, and interference path length dependence of the tunneling edge current ${ }^{29,53,63}$ that we denote as $F(T, V, L)$. The details of this edge physics can generally be complicated, but the most significant aspect is the coherence length and temperature, which is given as before in Eqs. (7) and (8), with the appropriate scaling exponents for excitations of a given state (which are given in the tables).

For the lowest order tunneling interference process, the braiding statistics contributes the factor ${ }^{34}$

$$
M_{a b}=\frac{S_{a b} S_{00}}{S_{0 a} S_{0 b}},
$$

where $S_{a b}$ is the topological $S$ matrix, and $a$ and $b$ are the topological charges of the tunneling edge excitation and the encircled bulk quasiparticle excitations, respectively. These combine to give the lowest order interference contribution to the tunneling current (in the asymptotic limit where the state of the bulk quasiparticles is projected onto a definite value of $b$ )

$$
I_{12}^{(\mathrm{qp})} \propto \operatorname{Re}\left\{e^{i 2 \pi e^{*} \Phi / e \Phi_{0}} M_{a b}^{*} F(T, V, L)\right\}
$$

If either $a$ or $b$ is an Abelian charge, $M_{a b}$ is simply a phase. More generally, when $a$ and $b$ are both non-Abelian charges, $M_{a b}$ is a complex number with $\left|M_{a b}\right| \leq 1$. This leads to the potential for a suppression of the interference term [Eq. (A2)] resulting from non-Abelian braiding statistics similar to the non-Abelian $\nu=5 / 2$ states.

For the non-Abelian FQH states considered here, the braiding statistics are essentially given by the $\mathrm{SU}(2)_{k}$ theories ${ }^{80}$ up to Abelian phase factors. These theories have topological charges $j=0,1 / 2,1, \ldots, k / 2$ and

$$
M_{j_{1} j_{2}}=\frac{\sin \left(\frac{\left(2 j_{1}+1\right)\left(2 j_{2}+1\right) \pi}{k+2}\right) \sin \left(\frac{\pi}{k+2}\right)}{\sin \left(\frac{\left(2 j_{1}+1\right) \pi}{k+2}\right) \sin \left(\frac{\left(2 j_{2}+1\right) \pi}{k+2}\right)} .
$$

The $k=2,3$, and 4 cases are the most pertinent to our discussion, so we write them out explicitly:

$$
M^{(2)}=\left[\begin{array}{ccc}
1 & 1 & 1 \\
1 & 0 & -1 \\
1 & -1 & 1
\end{array}\right] \text {, }
$$




$$
M^{(3)}=\left[\begin{array}{cccc}
1 & 1 & 1 & 1 \\
1 & \phi^{-2} & -\phi^{-2} & -1 \\
1 & -\phi^{-2} & -\phi^{-2} & 1 \\
1 & -1 & 1 & -1
\end{array}\right],
$$

where $\phi=\frac{1+\sqrt{5}}{2}$ is the Golden ratio, and

$$
M^{(4)}=\left[\begin{array}{ccccc}
1 & 1 & 1 & 1 & 1 \\
1 & \frac{1}{\sqrt{3}} & 0 & \frac{-1}{\sqrt{3}} & -1 \\
1 & 0 & \frac{1}{2} & 0 & 1 \\
1 & \frac{-1}{\sqrt{3}} & 0 & \frac{1}{\sqrt{3}} & -1 \\
1 & -1 & 1 & -1 & 1
\end{array}\right] .
$$

The braiding statistics of the MR, $\overline{\mathrm{Pf}}$, and $\mathrm{SU}(2)_{2} \mathrm{NAF}$ $\nu=5 / 2$ states are all derived from $\mathrm{SU}(2)_{2}$. The non-Abelian quasiparticles in these states carry $\mathrm{SU}(2)_{2}$ charge $1 / 2$. It follows that an odd number cluster of such quasiparticles will also carry a collective $\mathrm{SU}(2)_{2}$ charge of $1 / 2$, while an even number cluster will carry either 0 or 1 . Thus, looking at the $(j=1 / 2)$ middle column of Eq. (A4), we see exactly the source of the behavior described in Eq. (1).

\section{1. $\nu=7 / 3$}

For the $\nu=7 / 3 \mathrm{FQH}$ plateau, the leading candidates are the Laughlin (L) state, ${ }^{81}$ two types of Bonderson-Slingerland (BS) states, ${ }^{82}$ and a four-clustered Read-Rezayi (RR) state. ${ }^{75}$ (The bar indicates particle-hole conjugation.) The BS states considered here are hierarchically constructed over the MR and $\overline{\mathrm{Pf}}$ states, and so have similar non-Abelian statistics derived from Eq. (A4) using the fact that the non-Abelian quasiparticles carry $\mathrm{SU}(2)_{2}$ charge $1 / 2$. The $\mathrm{RR}_{k=4}$ state is related to $\mathrm{SU}(2)_{4}$, and so has more complicated non-Abelian statistics derived from Eq. (A6). Its fundamental $e / 6$ quasiparticles carry $\mathrm{SU}(2)_{4}$ charge $1 / 2$.

We see in Table III that all of these states have an $e / 3$ excitation with smallest scaling exponent $g=1 / 3$, and so one expects these to dominate the tunneling. The $\overline{\mathrm{RR}}_{k=4}$ state also has $e / 6$ excitations with $g=1 / 3$, which should give a comparable contribution to the tunneling current. The experiments of Refs. 22 and 43, which appear to observe $e / 3$ tunneling, but not $e / 6$ tunneling at $\nu=7 / 3$, seem to exclude the $\overline{\mathrm{RR}}_{k=4}$ state, while they agree with the $\mathrm{L}_{1 / 3}, \overline{\mathrm{BS}}_{2 / 3}$, and $\mathrm{BS}_{1 / 3}^{\psi}$ states. In fact, since the relevant excitations of these latter three states all have $e / 3$, and furthermore, the most relevant tunnelers are all Abelian, it will likely be difficult to distinguish between $\mathrm{L}_{1 / 3}, \overline{\mathrm{BS}}_{2 / 3}$, and $\mathrm{BS}_{1 / 3}^{\psi}$ using tunneling and interferometry experiments. Thermal transport experiments are probably the best hope of distinguishing between these.

\section{2. $\nu=12 / 5$}

For the $\nu=12 / 5 \mathrm{FQH}$ plateau, the leading candidates are the Haldane-Halperin (HH) state, ${ }^{83,84}$ two types of BS
TABLE III. Relevant quasiparticle excitations of model $\mathrm{FQH}$ states at $\nu=7 / 3$. Here we list their values of charge $e^{*}$; whether they are non-Abelian; their topological twist factor $\theta$; and their charge and neutral scaling exponents $g_{c}, g_{n}$, and $g$. The $\overline{\mathrm{BS}}, \mathrm{BS}^{\psi}$, and $\overline{\mathrm{RR}}_{k=4}$ states are non-Abelian, while the L state is Abelian. All of these have Abelian $e / 3$ Laughlin-type quasiparticles. (Note: The $e / 2$ excitation for $\overline{\mathrm{RR}}$ is marginal, but we include it for the sake of representing the possibility of $e / 2$ charge.)

\begin{tabular}{lcccccc}
\hline \hline$\nu=\frac{7}{3}$ & $e^{*}$ & $\mathrm{n}-\mathrm{A} ?$ & $\theta$ & $g_{c}$ & $g_{n}$ & $g$ \\
\hline $\mathrm{L}_{1 / 3}:$ & $e / 3$ & No & $e^{i \pi / 3}$ & $1 / 3$ & 0 & $1 / 3$ \\
\hline$\overline{\mathrm{BS}}_{2 / 3}:$ & $e / 3$ & Yes & $e^{-i 7 \pi / 24}$ & $1 / 3$ & $5 / 8$ & $23 / 24$ \\
& $e / 3$ & No & $e^{i \pi / 3}$ & $1 / 3$ & 0 & $1 / 3$ \\
\hline $\mathrm{BS}_{1 / 3}^{\psi}:$ & $e / 3$ & Yes & $e^{i 5 \pi / 24}$ & $1 / 3$ & $3 / 8$ & $17 / 24$ \\
& $e / 3$ & No & $e^{i \pi / 3}$ & $1 / 3$ & 0 & $1 / 3$ \\
$\overline{\mathrm{RR}}_{k=4}:$ & $e / 6$ & Yes & $e^{-i \pi / 6}$ & $1 / 12$ & $1 / 4$ & $1 / 3$ \\
& $e / 3$ & No & $e^{i \pi / 3}$ & $1 / 3$ & 0 & $1 / 3$ \\
& $e / 2$ & Yes & $e^{i \pi / 2}$ & $3 / 4$ & $1 / 4$ & 1 \\
\hline \hline
\end{tabular}

states, ${ }^{82}$ and a three-clustered RR state. ${ }^{75}$ These BS states again have non-Abelian statistics derived from Eq. (A4) using the fact that the non-Abelian quasiparticles carry $\mathrm{SU}(2)_{2}$ charge $1 / 2$. The $\mathrm{RR}_{k=3}$ state is related to $\mathrm{SU}(2)_{3}$, and so has non-Abelian statistics derived from Eq. (A5). Its fundamental $e / 5$ quasiparticles carry $\mathrm{SU}(2)_{3}$ charge $1 / 2$.

We see in Table IV that all of these states have an Abelian $2 e / 5$ excitation with scaling exponent $g=2 / 5$, so there should always be a background of such excitations in tunneling. The $\mathrm{HH}_{2 / 5}, \mathrm{BS}_{2 / 5}$, and $\overline{\mathrm{BS}}_{3 / 5}^{\psi}$ states all have an Abelian $e / 5$ excitation with $g=3 / 5$, so there should be a weaker background of these excitations in the tunneling. The smallest scaling exponent for the $\mathrm{BS}_{2 / 5}$ state belongs to the non-

TABLE IV. Relevant quasiparticle excitations of model FQH states at $\nu=12 / 5$. Here we list their values of charge $e^{*}$; whether they are non-Abelian; their topological twist factor $\theta$; and their charge and neutral scaling exponents $g_{c}, g_{n}$, and $g$. The $\mathrm{BS}, \overline{\mathrm{BS}}$, and $\overline{\mathrm{RR}}_{k=3}$ states are non-Abelian, while the $\mathrm{HH}$ state is Abelian. All of these have Abelian 2e/5 Laughlin-type quasiparticles; all of these except $\overline{\mathrm{RR}}$ have a relevant Abelian $e / 5$ quasiparticle.

\begin{tabular}{lcccccc}
\hline \hline$\nu=\frac{12}{5}$ & $e^{*}$ & $\mathrm{n}-\mathrm{A} ?$ & $\theta$ & $g_{c}$ & $g_{n}$ & $g$ \\
\hline $\mathrm{HH}_{2 / 5}:$ & $e / 5$ & $\mathrm{No}$ & $e^{i 3 \pi / 5}$ & $1 / 5$ & $2 / 5$ & $3 / 5$ \\
& $2 e / 5$ & $\mathrm{No}$ & $e^{i 2 \pi / 5}$ & $2 / 5$ & 0 & $2 / 5$ \\
\hline $\mathrm{BS}_{2 / 5}:$ & $e / 5$ & Yes & $e^{i 9 \pi / 40}$ & $1 / 10$ & $1 / 8$ & $9 / 40$ \\
& $e / 5$ & No & $e^{-i 2 \pi / 5}$ & $1 / 10$ & $1 / 2$ & $3 / 5$ \\
& $2 e / 5$ & No & $e^{i 2 \pi / 5}$ & $2 / 5$ & 0 & $2 / 5$ \\
$\overline{\mathrm{BS}}_{3 / 5}:$ & $e / 5$ & Yes & $e^{-i 11 \pi / 40}$ & $1 / 10$ & $3 / 8$ & $19 / 40$ \\
& $e / 5$ & No & $e^{-i 2 \pi / 5}$ & $1 / 10$ & $1 / 2$ & $3 / 5$ \\
& $2 e / 5$ & No & $e^{i 2 \pi / 5}$ & $2 / 5$ & 0 & $2 / 5$ \\
\hline$\overline{\mathrm{RR}}_{k=3}:$ & $e / 5$ & Yes & $e^{-i \pi / 5}$ & $1 / 10$ & $3 / 10$ & $2 / 5$ \\
& $2 e / 5$ & No & $e^{i 2 \pi / 5}$ & $2 / 5$ & 0 & $2 / 5$ \\
\hline \hline
\end{tabular}


Abelian $e / 5$ excitation, which is therefore expected to dominate the tunneling in this state. The $\overline{\mathrm{BS}}_{3 / 5}^{\psi}$ state has a nonAbelian $e / 5$ excitation which has slightly less relevant tunneling operator than the $2 e / 5$ excitation. The $\overline{\mathrm{RR}}_{k=3}$ state has a non-Abelian $e / 5$ excitation with the same scaling exponent $g=2 / 5$ as the $2 e / 5$ excitation, so they should have roughly equal contribution to tunneling.

In interferometry experiments, the $\mathrm{BS}_{2 / 5}, \overline{\mathrm{BS}}_{3 / 5}^{\psi}$, and $\overline{\mathrm{RR}}_{k=3}$ states will all exhibit $e / 5$ oscillations that will sometimes be suppressed. However, there are important distinctions within this behavior that can distinguish between them. In particular, the BS states will exhibit an even-odd effect similar to Eq. (1), always returning to suppression for $n_{q_{0}}$ odd, where $n_{q_{0}}$ is the number of non-Abelian $e / 5$ fundamental quasiparticles. On the other hand, the RR state can exhibit both suppression and full amplitude oscillations for all values of $n_{q}$ and it has a probability of switching between them when a given quasiparticle is taken in and out of the interferometry region. Furthermore, when the oscillations are suppressed for the BS state, the smaller amplitude $e / 5$ oscillations will be due to tunneling of the Abelian $e / 5$ excitations (which will always be present), because the nonAbelian excitation will have fully suppressed interference. The relative contribution to the tunneling of these excitations is not a fixed amount and will change depending on temperature and voltage (i.e., they have different scaling). In contrast to this, the suppression that would be observed in the RR state is due entirely to the braiding statistics of the nonAbelian $e / 5$ excitation and the suppressed oscillation amplitude should always be a constant factor of $\phi^{-2} \approx 0.38$ smaller than the full oscillation amplitude.

\section{3. $\nu=8 / 3$}

The candidates for the $\nu=8 / 3 \mathrm{FQH}$ plateau are, of course, similar to the $\nu=7 / 3$ candidates, since the filling fractions are particle-hole dual. We stress, however, that the physically observed states at these filling fractions need not be particlehole dual to each other, since physical effects, such as Landau level mixing, will tend to break particle-hole symmetry at these fillings. The leading candidates are the Laughlin state, ${ }^{81}$ two types of BS states, ${ }^{82}$ and a four-clustered RR state. $^{75}$ These BS states again have non-Abelian statistics derived from Eq. (A4) using the fact that the non-Abelian quasiparticles carry $\mathrm{SU}(2)_{2}$ charge $1 / 2$. The $\mathrm{RR}_{k=4}$ state again is related to $\mathrm{SU}(2)_{4}$, with non-Abelian statistics derived from Eq. (A6) and fundamental $e / 6$ quasiparticles carrying $\mathrm{SU}(2)_{4}$ charge $1 / 2$.

We see in Table $\mathrm{V}$ that all of these states have an Abelian $2 e / 3$ excitation with scaling exponent $g=2 / 3$, so there should always be a background of such excitations in tunneling. The $\overline{\mathrm{L}}_{1 / 3}, \mathrm{BS}_{2 / 3}$, and $\overline{\mathrm{BS}}_{1 / 3}^{\psi}$ states all have an $e / 3$ excitation also with $g=2 / 3$, so these two excitations are expected to have roughly equal contribution to the tunneling in these theories. However, the smallest scaling exponent for the $\mathrm{BS}_{2 / 3}$ and $\overline{\mathrm{BS}}_{1 / 3}^{\psi}$ states belong to non-Abelian $e / 3$ excitations, which are therefore expected to dominate the tunneling in these states. The smallest scaling exponent for the $\mathrm{RR}_{k=4}$ state belongs to the non-Abelian $e / 6$ excitation, which
TABLE V. Relevant quasiparticle excitations of model FQH states at $\nu=8 / 3$. Here we list their values of charge $e^{*}$; whether they are non-Abelian; their topological twist factor $\theta$; and their charge and neutral scaling exponents $g_{c}, g_{n}$, and $g$. The $\mathrm{BS}, \overline{\mathrm{BS}} \psi$, and $\mathrm{RR}_{k=4}$ states are non-Abelian, while the $\overline{\mathrm{L}}$ state is Abelian. All of these have Abelian 2e/3 Laughlin-type quasiparticles; all of these except RR have a relevant Abelian $e / 3$ quasiparticle.

\begin{tabular}{lcccccc}
\hline \hline$\nu=\frac{8}{3}$ & $e^{*}$ & $\mathrm{n}-\mathrm{A} ?$ & $\theta$ & $g_{c}$ & $g_{n}$ & $g$ \\
\hline$\overline{\mathrm{L}}_{1 / 3}:$ & $e / 3$ & $\mathrm{No}$ & $e^{-i \pi / 3}$ & $1 / 3$ & $1 / 3$ & $2 / 3$ \\
& $2 e / 3$ & No & $e^{i 2 \pi / 3}$ & $2 / 3$ & 0 & $2 / 3$ \\
\hline $\mathrm{BS}_{2 / 3}:$ & $e / 3$ & Yes & $e^{i 7 \pi / 24}$ & $1 / 6$ & $1 / 8$ & $7 / 24$ \\
& $e / 3$ & No & $e^{i 2 \pi / 3}$ & $1 / 3$ & $1 / 3$ & $2 / 3$ \\
& $2 e / 3$ & No & $e^{i 2 \pi / 3}$ & $2 / 3$ & 0 & $2 / 3$ \\
$\overline{\mathrm{BS}}_{1 / 3}:$ & $e / 3$ & Yes & $e^{-i 5 \pi / 24}$ & $1 / 6$ & $3 / 8$ & $13 / 24$ \\
& $e / 3$ & No & $e^{i 2 \pi / 3}$ & $1 / 6$ & $1 / 2$ & $2 / 3$ \\
& $2 e / 3$ & No & $e^{i 2 \pi / 3}$ & $2 / 3$ & 0 & $2 / 3$ \\
\hline $\mathrm{RR}_{k=4}:$ & $e / 6$ & Yes & $e^{i \pi / 6}$ & $1 / 24$ & $1 / 8$ & $1 / 6$ \\
& $e / 3$ & Yes & $e^{i \pi / 3}$ & $1 / 6$ & $1 / 6$ & $1 / 3$ \\
& $e / 2$ & Yes & $e^{i \pi / 2}$ & $3 / 8$ & $1 / 8$ & $1 / 2$ \\
& $2 e / 3$ & No & $e^{i 2 \pi / 3}$ & $2 / 3$ & 0 & $2 / 3$ \\
\hline \hline
\end{tabular}

should thus dominate tunneling. There are additional relevant tunnelers for $\mathrm{RR}_{k=4}$ that are non-Abelian with different statistics than the fundamental quasiparticle, namely, the $e / 3$ and $e / 2$ excitations which carry $\mathrm{SU}(2)_{4}$ charges 1 and $3 / 2$, respectively. The experiments of Ref. 22, which observes only $e / 3$ tunneling, appear to exclude the $\mathrm{RR}_{k=4}$ state and best agree with the $\mathrm{BS}_{2 / 3}$ and $\overline{\mathrm{BS}}_{1 / 3}^{\psi}$ states.

\section{4. $\nu=14 / 5$}

The $\nu=14 / 5 \mathrm{FQH}$ plateau is most likely the standard (particle-hole conjugate) Laughlin state,${ }^{81}$ but we include this filling fraction for completeness and list BS states ${ }^{82}$ as an (unlikely) alternative candidate. These BS states again have non-Abelian statistics derived from Eq. (A4) using the fact that the non-Abelian quasiparticles carry $\mathrm{SU}(2)_{2}$ charge $1 / 2$.

We see in Table VI that all of these states have Abelian $e / 5$ and $4 e / 5$ excitations with relevant scaling exponents, so there should always be a background of such excitations in tunneling. The $e / 5$ excitation is the most relevant tunneler for the $\overline{\mathrm{L}}_{1 / 5}$ state. For the $\mathrm{BS}_{4 / 5}$ and $\overline{\mathrm{BS}}_{1 / 5}^{\psi}$ states, the nonAbelian $2 e / 5$ excitation has the most relevant tunneling.

\section{5. $\nu=19 / 8$}

While a fully developed FQH plateau has not been observed at $\nu=19 / 8$, there does appear to be a feature of a developing plateau there, ${ }^{78,79}$ and it is the next filling fraction in the sequence of BS states following $\nu=12 / 5$, so we will list these states. ${ }^{82}$ These BS states again have non-Abelian statistics derived from Eq. (A4) using the fact that the nonAbelian quasiparticles carry $\mathrm{SU}(2)_{2}$ charge $1 / 2$.

We see in Table VII that for both of these states the two smallest tunneling exponents belong to a non-Abelian $3 e / 16$ 
TABLE VI. Relevant quasiparticle excitations of model FQH states at $\nu=14 / 5$. Here we list their values of charge $e^{*}$; whether they are non-Abelian; their topological twist factor $\theta$; and their charge and neutral scaling exponents $g_{c}, g_{n}$, and $g$. The BS and $\overline{\mathrm{BS}}_{1 / 5}^{\psi}$ states are non-Abelian, while the $\overline{\mathrm{L}}$ state is Abelian. All of these have Abelian 4e/5 Laughlin-type quasiparticles.

\begin{tabular}{lcccccc}
\hline \hline$\nu=\frac{14}{5}$ & $e^{*}$ & $\mathrm{n}-\mathrm{A} ?$ & $\theta$ & $g_{c}$ & $g_{n}$ & $g$ \\
\hline$\overline{\mathrm{L}}_{1 / 5}:$ & $e / 5$ & No & $e^{-i \pi / 5}$ & $1 / 20$ & $1 / 4$ & $3 / 10$ \\
& $3 e / 5$ & No & $e^{i \pi / 5}$ & $9 / 20$ & $1 / 4$ & $7 / 10$ \\
& $4 e / 5$ & No & $e^{i 4 \pi / 5}$ & $4 / 5$ & 0 & $4 / 5$ \\
\hline $\mathrm{BS}_{4 / 5}:$ & $e / 5$ & No & $e^{i 4 \pi / 5}$ & $1 / 20$ & $3 / 4$ & $4 / 5$ \\
& $e / 5$ & Yes & $e^{i 37 \pi / 40}$ & $1 / 20$ & $7 / 8$ & $37 / 40$ \\
& $2 e / 5$ & Yes & $e^{i 13 \pi / 40}$ & $1 / 5$ & $1 / 8$ & $13 / 40$ \\
& $4 e / 5$ & No & $e^{i 4 \pi / 5}$ & $4 / 5$ & 0 & $4 / 5$ \\
\hline$\overline{\mathrm{BS}}_{1 / 5}^{\psi}:$ & $e / 5$ & No & $e^{i 4 \pi / 5}$ & $1 / 20$ & $3 / 4$ & $4 / 5$ \\
& $e / 5$ & Yes & $e^{i 17 \pi / 40}$ & $1 / 20$ & $5 / 8$ & $27 / 40$ \\
& $2 e / 5$ & Yes & $e^{i 7 \pi / 40}$ & $1 / 5$ & $3 / 8$ & $23 / 40$ \\
& $4 e / 5$ & No & $e^{i 4 \pi / 5}$ & $4 / 5$ & 0 & $4 / 5$ \\
\hline \hline
\end{tabular}

excitation and an Abelian $3 e / 8$ excitation. The scaling exponent of the Abelian $3 e / 8$ excitation is $g=3 / 8$ in both theories, while the non-Abelian $3 e / 16$ excitation has tunneling exponent $g=7 / 32$ for the BS state and $g=15 / 32$ for the $\overline{B S}^{\psi}$ state. Thus, the contribution of the non-Abelian $3 e / 16$ excitation will be slightly stronger than that of the Abelian $3 e / 8$ excitation for the BS state, whereas it will be the other way around for the $\overline{\mathrm{BS}^{\psi}}$ state.

We also mention that a BS-type hierarchy could be built over the $\mathrm{SU}(2)_{2} \mathrm{NAF}$ state to produce candidates for all the filling fractions listed above. ${ }^{82}$ The relevant data could be read off the above tables for the non-Abelian quasiparticle excitations in the BS states by simply adding $1 / 4$ to $g_{n}$ and $g$ and multiplying the twist factors by $e^{i \pi / 4}$.

TABLE VII. Relevant quasiparticle excitations of model FQH states at $\nu=19 / 8$. Here we list their values of charge $e^{*}$; whether they are non-Abelian; their topological twist factor $\theta$; and their charge and neutral scaling exponents $g_{c}, g_{n}$, and $g$. The BS and $\overline{\mathrm{BS}^{\psi}} \psi$ states are non-Abelian. Both of these have Abelian $3 e / 8$ Laughlintype quasiparticles.

\begin{tabular}{ccccccc}
\hline \hline$\nu=\frac{19}{8}$ & $e^{*}$ & $\mathrm{n}-\mathrm{A} ?$ & $\theta$ & $g_{c}$ & $g_{n}$ & $g$ \\
\hline $\mathrm{BS}_{3 / 8}:$ & $e / 16$ & Yes & $e^{-i 17 \pi / 32}$ & $1 / 96$ & $19 / 24$ & $77 / 96$ \\
& $e / 8$ & No & $e^{-i 5 \pi / 8}$ & $1 / 24$ & $2 / 3$ & $17 / 24$ \\
& $3 e / 16$ & Yes & $e^{i 7 \pi / 32}$ & $3 / 32$ & $1 / 8$ & $7 / 32$ \\
& $e / 4$ & No & $e^{-i \pi / 2}$ & $1 / 6$ & $2 / 3$ & $5 / 6$ \\
& $3 e / 8$ & No & $e^{i 3 \pi / 8}$ & $3 / 8$ & 0 & $3 / 8$ \\
\hline$\overline{\mathrm{BS}}_{5 / 8}^{\psi}:$ & $e / 8$ & No & $e^{-i 5 \pi / 8}$ & $1 / 24$ & $2 / 3$ & $17 / 24$ \\
& $3 e / 16$ & Yes & $e^{-i 9 \pi / 32}$ & $3 / 32$ & $3 / 8$ & $15 / 32$ \\
& $e / 4$ & No & $e^{-i \pi / 2}$ & $1 / 6$ & $2 / 3$ & $5 / 6$ \\
& $3 e / 8$ & No & $e^{i 3 \pi / 8}$ & $3 / 8$ & 0 & $3 / 8$ \\
\hline \hline
\end{tabular}

\section{APPENDIX B: CHARGE $e / 4$ AND $e / 2$ BACKSCATTERING MATRIX ELEMENTS}

In this section, we examine the tunneling amplitudes of $e / 4$ and $e / 2$ quasiparticles in detail and argue that generically $\Gamma_{e / 4} \gg \Gamma_{e / 2}$. When they are small, the tunneling amplitudes $\Gamma_{e / 4}$ and $\Gamma_{e / 2}$ are the matrix elements for the transfer of charge from one edge of a Hall device to the other. For simplicity and concreteness, let us suppose that the device is a Hall bar with a single constriction. Then, the transfer of charge $q$ from one edge to the other entails a momentum change $\Delta k_{x} \sim(q / e) \Delta y / \ell_{0}^{2}$, where the $x$ direction is along the Hall bar. (This is seen most easily in Landau gauge, as we discuss below in the context of specific trial wave functions.) However, in order to cause a momentum change of $\Delta k_{x}$, the potential due to the gates must have weight at this wave vector; i.e., the matrix element is determined by the variation in the potential on a length scale $\Delta x \sim 1 / \Delta k_{x} \sim(e / q) \ell_{0}^{2} / \Delta y$. Hence, in order to transfer charge $q$ from one edge to the other, we need the potential to vary on a length scale $\Delta x$ satisfying

$$
\Delta x \cdot \Delta y \sim(e / q) \ell_{0}^{2} .
$$

If the constriction was much smaller than this, then we would expect that the potential would have comparable weight at the wave vector necessary for charge $e / 4$ transfer, $\Delta k_{x} \sim \Delta y / 4 \ell_{0}^{2}$, and at the larger wave vector necessary for charge $e / 2$ transfer, $\Delta k_{x} \sim \Delta y / 2 \ell_{0}^{2}$. Otherwise, we expect the weight to fall off rapidly with the wave vector and to have $\Gamma_{e / 4} \gg \Gamma_{e / 2}$.

This can be made a little more precise by considering, for the sake of concreteness, the MR Pfaffian state. We work in Landau gauge on a cylinder: ${ }^{85}$

$$
\Psi_{0}=S\left(Z_{1}, \ldots, Z_{n}\right) \operatorname{Pf}\left(\frac{1}{Z_{i}-Z_{j}}\right) \prod_{i>j}\left(Z_{i}-Z_{j}\right)^{2} e^{\Sigma_{i} y_{i}^{2} / 2 \ell_{0}^{2}},
$$

where $Z_{i}=e^{i\left(x_{i}+i y_{i}\right) / r}, x_{i}$ and $y_{i}$ are the coordinates around and along the cylinder, respectively, and $r$ is the radius of the cylinder. $S\left(Z_{1}, \ldots, Z_{n}\right)$ is a symmetric polynomial which deforms the shape of the Hall droplet from a rotationally symmetric band around the cylinder to one with a constriction. For instance, we could take $S\left(Z_{1}, \ldots, Z_{n}\right)=\Pi_{i}\left(Z_{i}-\zeta_{1}\right)^{p}\left(Z_{i}\right.$ $\left.-\zeta_{2}\right)^{p}$ where $\zeta_{1}$ and $\zeta_{2}$ are points outside the droplet with the same $x$ coordinate. The precise form of $S\left(Z_{1}, \ldots, Z_{n}\right)$ is not important at the present level of discussion, but we will assume that it is a polynomial of degree $q$ which is less than $2 N_{e}$. Then, the wave function

$$
\Psi_{1 / 4}=S\left(Z_{1}, \ldots, Z_{n}\right) \operatorname{Pf}\left(\frac{Z_{i}+Z_{j}}{Z_{i}-Z_{j}}\right) \prod_{i>j}\left(Z_{i}-Z_{j}\right)^{2} e^{\Sigma_{i} y_{i}^{2} / 2 \ell_{0}^{2}}
$$

has charge $e / 4$ transferred from one edge to the other, while

$$
\Psi_{1 / 2}=S\left(Z_{1}, \ldots, Z_{n}\right) \prod_{i} Z_{i} \operatorname{Pf}\left(\frac{1}{Z_{i}-Z_{j}}\right) \prod_{i>j}\left(Z_{i}-Z_{j}\right)^{2} e^{\Sigma_{i} y_{i}^{2} / 2 \ell_{0}^{2}}
$$

has charge $e / 2$ transferred from one edge to the other.

The tunneling matrix elements $\Gamma_{e / 4}$ and $\Gamma_{e / 2}$ for charge- $e / 4$ and $e / 2$ quasiparticles, respectively, are 


$$
\Gamma_{e / 4}=\left\langle\Psi_{1 / 4}|\hat{V}| \Psi_{0}\right\rangle, \quad \Gamma_{e / 2}=\left\langle\Psi_{1 / 2}|\hat{V}| \Psi_{0}\right\rangle,
$$

where

$$
\hat{V}=\int d x d y V(x, y) \sum_{i} \delta^{(2)}\left(z-z_{i}\right)
$$

and $V(x, y)$ is the potential due to the gates which define the point contact.

While we would need a detailed knowledge of $V(x, y)$ and of the precise shape of the Hall droplet in order to determine $\Gamma_{e / 4}$ and $\Gamma_{e / 2}$ quantitatively, we can make a few qualitative remarks which echo our earlier observations. First, let us ignore $S\left(Z_{1}, \ldots, Z_{n}\right)$. Then, $\Psi_{0}, \Psi_{1 / 4}$, and $\Psi_{1 / 2}$ are eigenstates of angular momentum around the cylinder with eigenvalues $M=M_{0}, M_{0}+N / 2$, and $M_{0}+N$. Thus, the tunneling matrix elements $\Gamma_{e / 4}$ and $\Gamma_{e / 2}$ are controlled by $\tilde{V}\left(k_{x}, y\right)$ for $k_{x}=N / 2 r$ and $k_{x}=N / r$, respectively. These will be compa- rable if the scale $\Delta x$ over which the potential varies in the $x$ direction is smaller than $1 / k_{x} \sim r / N$. But the distance between the two edges $\Delta y$ is $\Delta y \sim \ell_{0}^{2} N / r$. Hence, we need $\Delta x \cdot \Delta y \sim \ell_{0}^{2}$ in order for the two tunneling matrix elements to be comparable. Otherwise, both are determined by the tails of the (Fourier transform of the) potential and $\Gamma_{e / 4} \gg \Gamma_{e / 2}$. The presence of the constrictions, which is reflected in $S\left(Z_{1}, \ldots, Z_{n}\right)$, means that the wave functions are no longer angular momentum eigenstates. Instead, $\Psi_{0}$ has nonzero amplitude for a range of angular momenta $M_{0}<M<M_{0}+m$ while $\Psi_{1 / 4}$ has nonzero amplitude for a range $M_{0}+N / 2$ $<M<M_{0}+N / 2+m$, and similarly for $\Psi_{1 / 2}$. Here, $m$ is determined by $S\left(Z_{1}, \ldots, Z_{n}\right)$; the minimum distance between the two edges at the constriction is $\Delta y \sim \ell_{0}^{2}(N-m) / r$. Thus, the tunneling matrix elements $\Gamma_{e / 4}$ and $\Gamma_{e / 2}$ are controlled by $k_{x}=(N-2 m) / 2 r$ and $k_{x}=(N-m) / r$. Hence, we obtain the same requirement as above, but with $\Delta y$ now understood as the distance between the two edges at their point of closest approach.
${ }^{1}$ R. Willett, J. P. Eisenstein, H. L. Stormer, D. C. Tsui, A. C. Gossard, and J. H. English, Phys. Rev. Lett. 59, 1776 (1987).

${ }^{2}$ W. Pan, J.-S. Xia, V. Shvarts, D. E. Adams, H. L. Stormer, D. C. Tsui, L. N. Pfeiffer, K. W. Baldwin, and K. W. West, Phys. Rev. Lett. 83, 3530 (1999).

${ }^{3}$ M. Greiter, X. G. Wen, and F. Wilczek, Nucl. Phys. B 374, 567 (1992).

${ }^{4}$ G. Moore and N. Read, Nucl. Phys. B 360, 362 (1991).

${ }^{5}$ C. Nayak and F. Wilczek, Nucl. Phys. B 479, 529 (1996).

${ }^{6}$ N. Read and E. Rezayi, Phys. Rev. B 54, 16864 (1996).

${ }^{7}$ J. M. Leinaas and J. Myrheim, Nuovo Cim., B 37, 1 (1977).

${ }^{8}$ G. A. Goldin, R. Menikoff, and D. H. Sharp, Phys. Rev. Lett. 54, 603 (1985).

${ }^{9}$ K. Fredenhagen, K. H. Rehren, and B. Schroer, Commun. Math. Phys. 125, 201 (1989).

${ }^{10}$ T. D. Imbo, C. S. Imbo, and E. C. G. Sudarshan, Phys. Lett. B 234, 103 (1990).

${ }^{11}$ J. Fröhlich and F. Gabbiani, Rev. Math. Phys. 2, 251 (1990).

${ }^{12}$ T. D. Imbo and J. March-Russell, Phys. Lett. B 252, 84 (1990).

${ }^{13}$ F. A. Bais, P. van Driel, and M. de Wild Propitius, Phys. Lett. B 280, 63 (1992)

${ }^{14}$ A. Y. Kitaev, Ann. Phys. 303, 2 (2003).

${ }^{15} \mathrm{~J}$. Preskill, in Introduction to Quantum Computation, edited by H.-K. Lo, S. Popescu, and T. P. Spiller (World Scientific, Singapore, 1998).

${ }^{16}$ M. H. Freedman, Proc. Natl. Acad. Sci. U.S.A. 95, 98 (1998).

${ }^{17}$ M. H. Freedman, M. J. Larsen, and Z. Wang, Commun. Math. Phys. 227, 605 (2002).

${ }^{18}$ M. H. Freedman, M. J. Larsen, and Z. Wang, Commun. Math. Phys. 228, 177 (2002).

${ }^{19}$ M. H. Freedman, A. Kitaev, M. J. Larsen, and Z. Wang, Bull., New Ser., Am. Math. Soc. 40, 31 (2002).

${ }^{20}$ J. Preskill, lecture notes, http://www.theory.caltech.edu/preskill/ ph219/topological.ps (2004).

${ }^{21}$ C. Nayak, S. H. Simon, A. Stern, M. Freedman, and S. Das Sarma, Rev. Mod. Phys. 80, 1083 (2008).
${ }^{22}$ M. Dolev, M. Heiblum, V. Umansky, A. Stern, and D. Mahalu, Nature (London) 452, 829 (2008).

${ }^{23}$ I. P. Radu, J. B. Miller, C. M. Marcus, M. A. Kastner, L. N. Pfeiffer, and K. W. West, Science 320, 899 (2008).

${ }^{24}$ S.-S. Lee, S. Ryu, C. Nayak, and M. P. A. Fisher, Phys. Rev. Lett. 99, 236807 (2007).

${ }^{25}$ M. Levin, B. I. Halperin, and B. Rosenow, Phys. Rev. Lett. 99, 236806 (2007)

${ }^{26}$ X. G. Wen, Phys. Rev. Lett. 66, 802 (1991).

${ }^{27}$ B. Blok and X. G. Wen, Nucl. Phys. B 374, 615 (1992).

${ }^{28}$ B. I. Halperin, Helv. Phys. Acta 56, 75 (1983).

${ }^{29}$ C. de C. Chamon, D. E. Freed, S. A. Kivelson, S. L. Sondhi, and X. G. Wen, Phys. Rev. B 55, 2331 (1997).

${ }^{30}$ E. Fradkin, C. Nayak, A. Tsvelik, and F. Wilczek, Nucl. Phys. B 516, 704 (1998).

${ }^{31}$ S. Das Sarma, M. Freedman, and C. Nayak, Phys. Rev. Lett. 94, 166802 (2005).

${ }^{32}$ A. Stern and B. I. Halperin, Phys. Rev. Lett. 96, 016802 (2006).

${ }^{33}$ P. Bonderson, A. Kitaev, and K. Shtengel, Phys. Rev. Lett. 96, 016803 (2006)

${ }^{34}$ P. Bonderson, K. Shtengel, and J. K. Slingerland, Phys. Rev. Lett. 97, 016401 (2006).

${ }^{35}$ P. Bonderson, M. Freedman, and C. Nayak, Phys. Rev. Lett. 101, 010501 (2008).

${ }^{36}$ P. Bonderson, M. Freedman, and C. Nayak, Ann. Phys. 324, 787 (2009).

${ }^{37}$ Y. Ji, Y. Chung, D. Sprinzak, M. Heiblum, D. Mahalu, and H Shtrikman, Nature (London) 422, 415 (2003).

${ }^{38}$ Y. Zhang, D. T. McClure, E. M. Levenson-Falk, C. M. Marcus, L. N. Pfeiffer, and K. W. West, Phys. Rev. B 79, 241304(R) (2009).

${ }^{39}$ F. E. Camino, W. Zhou, and V. J. Goldman, Phys. Rev. B 72, 075342 (2005)

${ }^{40}$ F. E. Camino, W. Zhou, and V. J. Goldman, Phys. Rev. Lett. 98, 076805 (2007).

${ }^{41}$ M. D. Godfrey, P. Jiang, W. Kang, S. H. Simon, K. W. Baldwin, 
L. N. Pfeiffer, and K. W. West, arXiv:0708.2448 (unpublished).

${ }^{42}$ R. L. Willett, M. J. Manfra, L. N. Pfeiffer, and K. W. West, arXiv:0807.0221v1 (unpublished).

${ }^{43}$ R. L. Willett, L. N. Pfeiffer, and K. W. West, Proc. Natl. Acad. Sci. U.S.A. 106, 8853 (2009).

${ }^{44}$ R. L. Willett, L. N. Pfeiffer, and K. W. West (unpublished).

${ }^{45}$ L. Venema, NATNEWS 452, 803 (2008).

${ }^{46}$ P. Bonderson, K. Shtengel, and J. K. Slingerland, Phys. Rev. Lett. 98, 070401 (2007).

${ }^{47}$ E. Grosfeld, S. H. Simon, and A. Stern, Phys. Rev. Lett. 96, 226803 (2006).

${ }^{48}$ P. H. Bonderson, Ph.D. thesis, Caltech, 2007.

${ }^{49}$ P. Bonderson, K. Shtengel, and J. K. Slingerland, Ann. Phys. 323, 2709 (2008).

${ }^{50}$ P. Fendley, M. P. A. Fisher, and C. Nayak, Phys. Rev. B 75, 045317 (2007).

${ }^{51}$ W. Bishara and C. Nayak, Phys. Rev. B 80, 155304 (2009).

${ }^{52}$ B. Rosenow, B. I. Halperin, S. H. Simon, and A. Stern, Phys. Rev. B 80, 155305 (2009).

${ }^{53}$ W. Bishara and C. Nayak, Phys. Rev. B 77, 165302 (2008).

${ }^{54}$ L. Fidkowski, arXiv:0704.3291 (unpublished).

${ }^{55}$ E. Ardonne and E.-A. Kim, J. Stat. Mech. (2008) L04001.

${ }^{56}$ X. Wan, Z.-X. Hu, E. H. Rezayi, and K. Yang, Phys. Rev. B 77, 165316 (2008).

${ }^{57}$ R. Ilan, E. Grosfeld, and A. Stern, Phys. Rev. Lett. 100, 086803 (2008).

${ }^{58}$ P. Bonderson, C. Nayak, and K. Shtengel, arXiv:0909.1056 (unpublished).

${ }^{59}$ J. A. Folk, S. R. Patel, S. F. Godijn, A. G. Huibers, S. M. Cronenwett, C. M. Marcus, K. Campman, and A. C. Gossard, Phys. Rev. Lett. 76, 1699 (1996).

${ }^{60}$ N. Ofek, M. Heiblum, V. Umansky, A. Stern, and D. Mahalu (unpublished).

${ }^{61}$ B. Rosenow and B. I. Halperin, Phys. Rev. Lett. 98, 106801 (2007).

${ }^{62}$ P. Fendley, M. P. A. Fisher, and C. Nayak, Phys. Rev. Lett. 97, 036801 (2006).

${ }^{63}$ X. G. Wen, Int. J. Mod. Phys. B 6, 1711 (1992).

${ }^{64}$ J. Martin, S. Ilani, B. Verdene, J. Smet, V. Umansky, D. Mahalu, D. Schuh, G. Abstreiter, and A. Yacoby, Science 305, 980 (2004).

${ }^{65}$ R. H. Morf, Phys. Rev. Lett. 80, 1505 (1998).
${ }^{66}$ E. H. Rezayi and F. D. M. Haldane, Phys. Rev. Lett. 84, 4685 (2000).

${ }^{67}$ A. E. Feiguin, E. Rezayi, C. Nayak, and S. Das Sarma, Phys. Rev. Lett. 100, 166803 (2008).

${ }^{68}$ G. Möller and S. H. Simon, Phys. Rev. B 77, 075319 (2008).

${ }^{69}$ M. R. Peterson, T. Jolicoeur, and S. Das Sarma, Phys. Rev. Lett. 101, 016807 (2008).

${ }^{70}$ A. E. Feiguin, E. Rezayi, K. Yang, C. Nayak, and S. Das Sarma, Phys. Rev. B 79, 115322 (2009).

${ }^{71}$ M. R. Peterson, K. Park, and S. Das Sarma, Phys. Rev. Lett. 101, 156803 (2008).

${ }^{72}$ R. H. Storni, R. H. Morf, and S. Das Sarma, arXiv:0812.2691 (unpublished).

${ }^{73}$ N. d'Ambrumenil and A. M. Reynolds, J. Phys. C 21, 119 (1988).

${ }^{74}$ M. R. Peterson, T. Jolicoeur, and S. Das Sarma, Phys. Rev. B 78, 155308 (2008).

${ }^{75}$ N. Read and E. Rezayi, Phys. Rev. B 59, 8084 (1999).

${ }^{76}$ E. H. Rezayi and N. Read, Phys. Rev. B 79, 075306 (2009).

${ }^{77}$ P. Bonderson, A. E. Feiguin, G. Möller, and J. K. Slingerland, arXiv:0901.4965 (unpublished).

${ }^{78}$ J. S. Xia, W. Pan, C. L. Vicente, E. D. Adams, N. S. Sullivan, H. L. Stormer, D. C. Tsui, L. N. Pfeiffer, K. W. Baldwin, and K. W. West, Phys. Rev. Lett. 93, 176809 (2004).

${ }^{79}$ W. Pan, J. S. Xia, H. L. Stormer, D. C. Tsui, C. Vicente, E. D. Adams, N. S. Sullivan, L. N. Pfeiffer, K. W. Baldwin, and K. W. West, Phys. Rev. B 77, 075307 (2008).

${ }^{80}$ E. Witten, Commun. Math. Phys. 121, 351 (1989).

${ }^{81}$ R. B. Laughlin, Phys. Rev. Lett. 50, 1395 (1983).

${ }^{82}$ P. Bonderson and J. K. Slingerland, Phys. Rev. B 78, 125323 (2008).

${ }^{83}$ F. D. M. Haldane, Phys. Rev. Lett. 51, 605 (1983).

${ }^{84}$ B. I. Halperin, Phys. Rev. Lett. 52, 1583 (1984).

${ }^{85}$ M. Milovanovic and N. Read, Phys. Rev. B 53, 13559 (1996).

${ }^{86}$ The edge mode velocities are not universal quantities and will generally be different for different samples, filling fractions, topological orders, etc. Lacking better physical estimates of the relevant velocities, we use these numerical velocity estimates to produce coherence length and temperature estimates for all the candidate $\nu=5 / 2$ states.

87 "With luck, we might see a topological qubit within a year." attributed to Kirill Shtengel, January 9, 2009. 